\title{
An Evacuation Model for Passenger Ships That Includes the Influence of Obstacles in Cabins
}

\author{
Baocheng Ni, ${ }^{1}$ Zhen $\mathrm{Li}^{2}{ }^{2}$ Pei Zhang, ${ }^{3}$ and Xiang $\mathrm{Li}^{4}$ \\ ${ }^{1}$ College of Ship Building, Harbin Engineering University, Harbin, China \\ ${ }^{2}$ College of Mechanical and Electrical Engineering, Harbin Engineering University, Harbin, China \\ ${ }^{3}$ Shanghai Merchant Ship Design and Research Institute, Shanghai, China \\ ${ }^{4}$ Dalian Neusoft University of Information, Dalian, China \\ Correspondence should be addressed to Baocheng Ni; nibaocheng@hrbeu.edu.cn
}

Received 28 February 2017; Revised 19 August 2017; Accepted 18 September 2017; Published 26 October 2017

Academic Editor: Mirko Viroli

Copyright (c) 2017 Baocheng Ni et al. This is an open access article distributed under the Creative Commons Attribution License, which permits unrestricted use, distribution, and reproduction in any medium, provided the original work is properly cited.

\begin{abstract}
Passenger behavior and ship environment are the key factors affecting evacuation efficiency. However, current studies ignore the interior layout of passenger ship cabins and treat the cabins as empty rooms. To investigate the influence of obstacles (e.g., tables and stools) on cabin evacuation, we propose an agent-based social force model for advanced evacuation analysis of passenger ships; this model uses a goal-driven submodel to determine a plan and an extended social force submodel to govern the movement of passengers. The extended social force submodel considers the interaction forces between the passengers, crew, and obstacles and minimises the range of these forces to improve computational efficiency. We drew the following conclusions based on a series of evacuation simulations conducted in this study: (1) the proposed model endows the passenger with the behaviors of bypassing and crossing obstacles, (2) funnel-shaped exits from cabins can improve evacuation efficiency, and (3) as the exit angle increases, the evacuation time also increases. These findings offer ship designers some insight towards increasing the safety of large passenger ships.
\end{abstract}

\section{Introduction}

With recent developments in shipbuilding, many passenger ships are able to accommodate several thousands of people. Furthermore, due to a series of accidents, the safety of large passenger ships has become a worldwide concern. To address safety concerns, the Maritime Safety Committee (MSC) of the International Maritime Organization (IMO) now requires that evacuation simulations be conducted during the design process. This mandatory requirement is stated clearly in the IMO MSC/Circulation 1238 (Circ. 1238) Guidelines for Evacuation Analysis for New and Existing Passenger Ships [1]. These guidelines provide a simplified method as well as a more advanced one. The simplified method evaluates the evacuation time using relatively simple expressions, whereas the advanced method employs computer simulation to determine the evacuation time. Although it is not currently required, it is generally accepted that the advanced method will become mandatory in the near future.
Over the past two decades, a variety of evacuation models have been proposed to analyze the process of evacuation. These models have been applied to different areas of design, including architectural design [2-4], ship design $[5,6]$, train design $[7,8]$, and aircraft design [9]. One of the most popular discrete evacuation models is the cellular automaton [10-13], which has the advantage of high computational efficiency because it treats time and space as discrete variables. However, because of the discrete nature of the cellular automata, this model cannot precisely evaluate the evacuation process. The social force model [14-16] is a continuous model that assumes that individuals are subject to physical and social forces; this model can be extended to include various types of behaviors such as overtaking [17], waiting [18], counter-flow collision avoidance [19], and leader following [20]. However, this type of model is unsuited for large-scale computer simulations because the time required to calculate the interaction forces between agents increases in proportion to the square of the number of agents. Moreover, agent-based models [21-23], 
in which pedestrians are represented as autonomous agents with cognitive and decision-making abilities, have become increasingly popular with recent developments in artificial intelligence. The agent-based model can be combined with the social force model $[19,24]$ or with cellular automata $[25,26]$. Moreover, some researchers [27-30] coupled their model encompassing a floor field based operational level and an adaptive tactical level component. Andresen et al. [27, 28] endowed an agent with the ability of decision-making related to pedestrian route choices in presence of fire. Crociani et al. $[29,30]$ took into account the impact of congestion on path planning and computational cost as well. In a small scene, their model computes faster than real-time calculations.

As such, researchers are increasingly employing the advanced computer simulation method to analyze passenger ship evacuation. For example, $\mathrm{Ha}$ et al. [31] developed a cellular automata simulation model, which included individuals, crowds, and the counter-flow avoidance behavior of passengers on a passenger ship, and have verified their model through the eleven tests specified in Circ. 1238 of the IMO. Park et al. [32] further validated the above-mentioned model through the comparison of simulation data and experimental data collected from real evacuation trials conducted in two full-scale ships and reported that the model met all of the requirements of each data set. Yuan et al. [33] proposed an advanced evacuation analysis based on neighborhood particle swarm optimisation, which included individual attributes, neighborhood attributes, and social attributes. They also considered the impacts of the movement of the passenger ship on evacuation dynamics. Chen and Han [34] proposed a new multigrid model based on cellular automata, which considered quantitative exit attraction, trajectory attraction, and the interaction force between passengers and found that, compared with the traditional cellular automata model, their model enhanced the continuity of the passengers' trajectories and was more accurate. Cho et al. [35] presented a velocitybased evacuation model, in which the emergency behaviors of the individual and crowd were expressed as independent velocity vectors. Their model passed the eleven tests specified in Circ. 1238. However, the aforementioned studies are not completely realistic as they ignore the interior layout of the cabins and neglect the influence of cabin obstacles on the evacuation process.

Outside the ship building community, a number of studies have addressed the evacuation of rooms containing obstacles. Varas et al. [36] simulated the evacuation of a room containing fixed obstacles using a cellular automaton. They introduced a panic parameter, making the model nondeterministic, and conducted classroom simulations. Alizadeh [37] used a dynamic cellular automaton to simulate the evacuation of a restaurant. Aik and Choon [38] introduced a modified dynamic cellular automata model that was able to take account of the effects of human emotional responses and crowd density at the exits and applied it to both classroom and restaurant simulations. However, these investigations have not considered the effect that the presence of stools may have on evacuation.

In this work, we propose an agent-based social force model to investigate the influence of cabin obstacles (tables and stools) on the evacuation process. At a decision-making level, we adopt a goal-driven model, which works in a similar way to human deliberation in planning, instead of a traditional rule-based model. At the locomotion level, we utilize an extended social force model, which includes the interaction forces between the individual, crew, and obstacles, in order to govern the agents' movements. Furthermore, the range of influence of the interaction forces is reduced to keep the computational complexity within acceptable limits. Using simulation, we study whether the model was able to endow an agent with the behaviors of bypassing and crossing obstacles and whether obstacles always hinder the evacuation process.

\section{Model}

We developed an agent-based social force model that contains submodels to account for crowd movement, path planning, and goal-driven decision-making. The agent in our evacuation model represents a passenger on a passenger ship. The crowd-movement submodel governs the movement of the crowd that decides how to travel from position $\mathrm{A}$ to position B. The path planning submodel plans a shortest path from the agent's current position to its targeted position. The decision-making submodel decomposes abstract goals into subgoals to create a concrete plan that contains a series of targets.

2.1. Crowd-Movement Submodel. We combined the social force [15] and steering force [44] models to create our crowdmovement model. Social forces were used to influence the individual behavior of agents and steering forces to influence the group behavior of agents. This model assumes that each agent $i$ is affected by the desired force $\mathbf{f}_{i}^{\text {de }}$, the interaction force $\mathbf{f}_{i W}$ between agent $i$ and wall $W$, the interaction force $\mathbf{f}_{i j}$ between agent $i$ and $j$, and the resistance force $\mathbf{f}_{i S}^{\mathrm{f}}$ generated by stool $S$. Note that in the proposed model the influence of tables need not be explicitly considered since it treats a table as an area enclosed by four walls that the passenger cannot traverse.

The change of velocity $\mathbf{v}_{i}$ of an agent at time $t$ can be calculated by

$$
m_{i} \frac{\mathrm{d} \mathbf{v}_{i}}{\mathrm{~d} t}=\mathbf{f}_{i}^{\mathrm{de}}+\sum_{W} \mathbf{f}_{i W}+\sum_{\forall j \in B_{i}} \mathbf{f}_{i j}+\sum_{S} \mathbf{f}_{i S}^{\mathrm{f}},
$$

where $m_{i}$ is the mass of agent $i . B_{i}$ denotes the set of agents within the neighborhood radius $R$ of agent $i$ according to

$$
B_{i}=\left\{j \mid j \in A, j \neq i,\left\|\mathbf{p}_{i}-\mathbf{p}_{j}\right\|<R\right\},
$$

where $\mathbf{p}_{i}$ and $\mathbf{p}_{j}$ denote the position vectors of agents $i$ and $j$, respectively, and $A$ denotes the set of all agents. Figure 1 shows the neighborhood radius of an agent. The gray circle represents the neighborhood of the blue agent. The purple agents are considered to be neighbors of the blue agent while the green agents are not. It is worth noting that the traditional social force model considers all agents when calculating the 


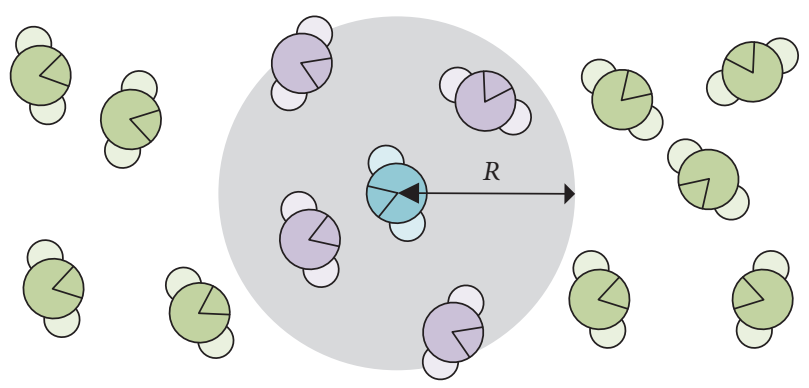

FIgURE 1: The neighborhood radius of an agent.

interaction force $\mathbf{f}_{i j}$ between the agents, which makes the simulation cumbersome and time-consuming since the time needed to calculate the interaction force between agents increases in proportion to the square of the number of agents. The current model only considers agents within the neighborhood radius $R$ when calculating the interaction force since the interaction forces beyond a certain distance $(>1 \mathrm{~m})$ are so small $(<1 \mathrm{~N})$ that they can be neglected.

The desired force $\mathbf{f}_{i}^{\mathrm{de}}$ reflects the behavior that agent $i$ attempts to move at a certain desired speed $v_{i}^{0}=\left|\mathbf{v}_{i}^{0}\right|$ towards a target specified by the decision-making model. The desired force is given by

$$
\mathbf{f}_{i}^{\mathrm{de}}=m_{i} \frac{\mathbf{v}_{i}^{0}(t)-\mathbf{v}_{i}(t)}{\tau_{i}}
$$

where $\tau_{i}$ is the characteristic time needed for agent $i$ to change his/her current velocity to the desired velocity. The desired velocity is given by

$$
\mathbf{v}_{i}^{0}(t)=v_{i}^{\max } \frac{\left(\mathbf{p}_{i}^{0}(t)-\mathbf{p}_{i}(t)\right)}{\left\|\mathbf{p}_{i}^{0}(t)-\mathbf{p}_{i}(t)\right\|},
$$

where $v_{i}^{\max }$ is the maximum walking speed of agent $i$ and the target position $\mathbf{p}_{i}^{0}(t)$ of agent $i$ is determined by the decisionmaking model.

The interaction force $\mathbf{f}_{i W}$ between agent $i$ and wall $W$ comprises three parts: the repulsive interaction force [14] $\mathbf{f}_{i W}^{\mathrm{r}}$ that maintains the agent at a certain distance away from the wall $W$, the body force [14] $\mathbf{f}_{i W}^{\mathrm{b}}$ that counteracts body compression, and the sliding friction force [14] $\mathbf{f}_{i W}^{\mathrm{s}}$ that impedes relative tangential motion if the agent touches the wall (see Figure 2). The interaction force between the agent and the wall is formulated according to (5)-(9) as follows:

$$
\begin{aligned}
\mathbf{f}_{i W} & =\mathbf{f}_{i W}^{\mathrm{r}}+\mathbf{f}_{i W}^{\mathrm{b}}+\mathbf{f}_{i W}^{\mathrm{s}}, \\
\mathbf{f}_{i W}^{\mathrm{r}} & =k_{\mathrm{r}} \frac{g\left(R-d_{i W}\right)}{R-r_{i}} \mathbf{n}_{i W}, \\
\mathbf{f}_{i W}^{\mathrm{b}} & =k_{\mathrm{b}} \exp \left(\frac{g\left(r_{i}-d_{i W}\right)}{B}\right) \mathbf{n}_{i W},
\end{aligned}
$$

$$
\begin{gathered}
\mathbf{f}_{i W}^{\mathrm{s}}=-k_{\mathrm{s}} g\left(r_{i}-d_{i W}\right)\left(\mathbf{v}_{i} \cdot \mathbf{t}_{i W}\right) \mathbf{t}_{i W}, \\
g(x)= \begin{cases}x & x>0 \\
0 & x \leq 0,\end{cases}
\end{gathered}
$$

where $B, k_{\mathrm{r}}, k_{\mathrm{b}}$, and $k_{\mathrm{s}}$ are constants, $r_{i}$ is the radius of agent $i, d_{i W}$ is the distance between agent $i$ and wall $W, R$ is the agent's neighborhood radius of the agent, $\mathbf{n}_{i W}$ denotes the normalised vector perpendicular to the wall $W$, and $\mathbf{t}_{i W}$ is the direction tangential the wall $W$. It is worth noting that $\mathbf{f}_{i W}^{\mathrm{r}}, \mathbf{f}_{i W}^{\mathrm{b}}$, and $\mathbf{f}_{i W}^{\mathrm{s}}$ are derived from the social force model. The range of influence of $\mathbf{f}_{i W}^{\mathrm{r}}$ is reduced compared to the traditional social force model in order to speed up the calculation; namely, the calculation only considers walls within the neighborhood of the agent.

The interaction force $\mathbf{f}_{i j}$ between agents $i$ and $j$ acting upon agent $i$ comprises five parts: the steering force of separation [44] $\mathbf{f}_{i j}^{\text {se }}$ that keeps an agent away from its neighbors (see Figure 3(a)), the steering force of cohesion [44] $\mathbf{f}_{i j}^{\text {co }}$ that keeps an agent close to the local group formed by his/her neighbors (steering the agent towards the center centroid of the geometrical shape formed by neighbors, see Figure 3(b)), the steering force of alignment [44] $\mathbf{f}_{i j}^{\text {al }}$ that matches the direction and speed of the agent's neighbors (see Figure 3(c)), the body force $\mathbf{f}_{i j}^{\mathrm{b}}$ that counteracts body compression, and the sliding friction force [14] $\mathbf{f}_{i j}^{\mathrm{s}}$ that impedes relative tangential motion in case agents touch each other (see Figure 4). The interaction force between two agents is formulated according to (10) as follows:

$$
\begin{aligned}
\mathbf{f}_{i j} & =\mathbf{f}_{i j}^{\mathrm{se}}+\mathbf{f}_{i j}^{\mathrm{co}}+\mathbf{f}_{i j}^{\mathrm{al}}+\mathbf{f}_{i j}^{\mathrm{b}}+\mathbf{f}_{i j}^{\mathrm{s}}, \\
\mathbf{f}_{i j}^{\mathrm{se}} & =k_{\mathrm{se}} \frac{\mathbf{n}_{i j}}{d_{i j}} \\
\mathbf{f}_{i j}^{\mathrm{co}} & =k_{\mathrm{co}} \frac{\mathbf{p}_{j}-\mathbf{p}_{i}}{\left|B_{i}\right|}, \\
\mathbf{f}_{i j}^{\mathrm{al}} & =k_{\mathrm{al}} \frac{\mathbf{v}_{j}-\mathbf{v}_{i}}{\left|B_{i}\right|}, \\
\mathbf{f}_{i j}^{\mathrm{b}} & =k_{\mathrm{b}} \exp \left(\frac{g\left(r_{i}-d_{i j}\right)}{B}\right) \mathbf{n}_{i j}, \\
\mathbf{f}_{i j}^{\mathrm{s}} & =k_{\mathrm{s}} g\left(r_{i}-d_{i j}\right) \Delta v_{j i}^{t} \mathbf{t}_{i j},
\end{aligned}
$$

where $d_{i j}=\left\|\mathbf{p}_{i}-\mathbf{p}_{j}\right\|$ is the distance between agent $i$ and $j, \mathbf{n}_{i j}=\left(n_{i j}^{1}, n_{i j}^{2}\right)=\left(\mathbf{p}_{i}-\mathbf{p}_{j}\right) / d_{i j}$ is the normalised vector pointing from agent $j$ to $i,\left|B_{i}\right|$ is the number of members of set $B_{i} . k_{\mathrm{se}}, k_{\mathrm{co}}$, and $k_{\mathrm{al}}$ are constants, $\mathbf{t}_{i j}=\left(-n_{i j}^{2}, n_{i j}^{1}\right)$ is the normalised vector perpendicular to $\mathbf{n}_{i j}$, and $\Delta v_{j i}^{t}=\left(\mathbf{v}_{j}-\mathbf{v}_{i}\right) \cdot \mathbf{t}_{i j}$ is the tangential velocity difference. It is worth noting that $\mathbf{f}_{i j}^{\mathrm{se}}, \mathbf{f}_{i j}^{\mathrm{co}}$, and $\mathbf{f}_{i j}^{\mathrm{al}}$ are derived from the steering force model and toggled by the decision-making model, whereas $\mathbf{f}_{i j}^{\mathrm{b}}$ and $\mathbf{f}_{i j}^{\mathrm{s}}$ are derived from the social force model and are analogous to $\mathbf{f}_{i W}^{b}$ and $\mathbf{f}_{i W}^{\mathrm{s}}$ in (5). 

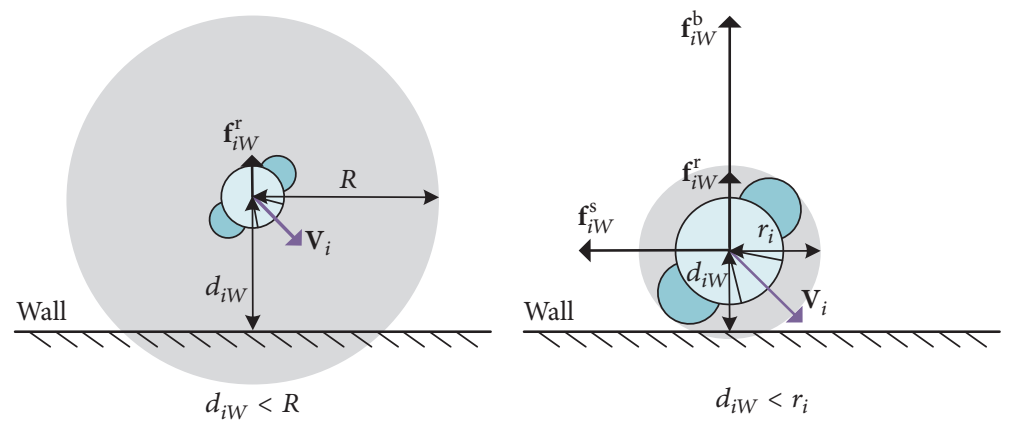

FIGURE 2: The interaction force between agent and wall.

To investigate this, our model introduced a resistance force simulating the role played by stools in an evacuation. The resistance force $\mathbf{f}_{i S}^{\mathrm{f}}$ from stool $S$ impeding the movement of agent $i$ is given by

$$
\mathbf{f}_{i S}^{\mathrm{f}}=-k_{\mathrm{f}} \frac{g\left(r_{i}+r_{S}^{\mathrm{st}}-d_{i S}\right)}{r_{i}+r_{S}^{\mathrm{st}}} \mathbf{v}_{i},
$$

where $k_{\mathrm{f}}$ is a constant, $r_{S}^{\text {st }}$ is the approximate radius of stool $S$, and $d_{i S}$ is the distance between agent $i$ and stool $S$ (see Figure 5).

Finally, the change in the position $\mathbf{p}_{i}(t)$ of agent $i$ is given by

$$
\frac{\mathrm{d} \mathbf{p}_{i}}{\mathrm{~d} t}=\mathbf{v}_{i}(t)
$$

2.2. Path Planning Submodel. We need to address the environmental representation issue before planning the shortest path towards an exit. The environment of the passenger ship is represented in the form of a graph $G(N, E)$ that contains all the locations (nodes; $N$ ) in the environment that agents may visit and all the connections (edges; $E$ ). We first set a single node called the seed node in the environment and then apply a breadth-first search to obtain all other nodes on the graph (see Figure 6(a)). The breadth-first algorithm searches for nodes from the seed in eight directions and adds nodes to the graph if they can be reached without penetrating a wall. In this model, the spacing between nodes is set to $0.4 \mathrm{~m}(2 \sqrt{2} / 5 \mathrm{~m}$ in the diagonal direction); however, the spacing can be changed depending on calculation accuracy and calculation time. Each edge of the graph contains the cost of traveling from one node to another.

Our path planning model planned the shortest path $P_{i j}$ (see Figure 6(b)) from the current position of agent $i$ to exit $j$ by Dijkstra search algorithm [46]. We run a single instance of the Dijkstra algorithm before a simulation setting each exit as a source node and storing parents for each node discovered. Then, we obtained the shortest-path tree of each exit. The shortest path can be easily obtained by starting from a node that is closest to an agent and repeatedly searching for the parent of the node until the parent node is an exit.

We used the horizontal distance (Euclidean distance) between nodes as the cost of the edges connecting those nodes with the exception of the edges near stools. To reflect the ability to bypass stools, we increased the costs of edges near stools to a larger value such that an agent will avoid these edges in searching for the shortest path to the exit. To modify these costs, we assigned different virtual heights to the nodes near each stool according to the total distance from the center of each stool, and then costs were calculated using a trigonometric function based on the horizontal distance and the virtual height. The cost $c_{N M}$ of edge $E_{M N}$ is given by

$$
\begin{aligned}
c_{N M} & =\sqrt{\left(h_{M}-h_{N}\right)^{2}+d_{N M}^{2},} \\
h_{N} & =\sum_{S} \frac{g\left(r+r_{S}^{\mathrm{st}}-d_{N S}\right)}{r+r_{S}^{\mathrm{st}}} h_{\max },
\end{aligned}
$$

where $h_{M}$ and $h_{N}$ are the virtual heights of nodes $N_{M}$ and $N_{N}$, respectively, $d_{N M}$ is the horizontal distance (Euclidean distance) between nodes $N_{N}$ and $N_{M}, r$ is the radius of the passenger, $d_{N S}$ is the horizontal distance (Euclidean distance) between node $N_{N}$ and stool $S$, and $h_{\max }$ is the maximum virtual height. Figure 7 presents an example of the calculation of the cost. The virtual height $h_{\max }$ is chosen to be $1.8 \mathrm{~m}$ such that agents prefer to bypass the stools. However, we could specify a different value for $h_{\max }$. If $h_{\max }$ is small, the agent might select a path that crosses the stools since the cost of that path can be lower than that of the path that bypasses the stools.

2.3. Goal-Driven Decision-Making Submodel. In some ways, a goal-driven decision-making model mirrors aspects of human deliberation in planning. Humans prefer to select higher-level abstract goals based on their desires and then to recursively break these goals down into a plan of action that can be executed without deliberation [47]. At each update, the agent evaluates its environmental state and chooses its most desired high-level goal, which the agent then breaks down into subgoals that are subsequently satisfied in turn.

Seven goals are predefined in our model: (1) evacuate, (2) evacuate individually, (3) evacuate by following the crowd, (4) plan path, (5) follow path, (6) move to node, and (7) enter assembly station. Goals (3), (6), and (7) are prime goals that cannot be broken down, whereas the others are composite goals that are comprised of several subgoals. At the beginning 

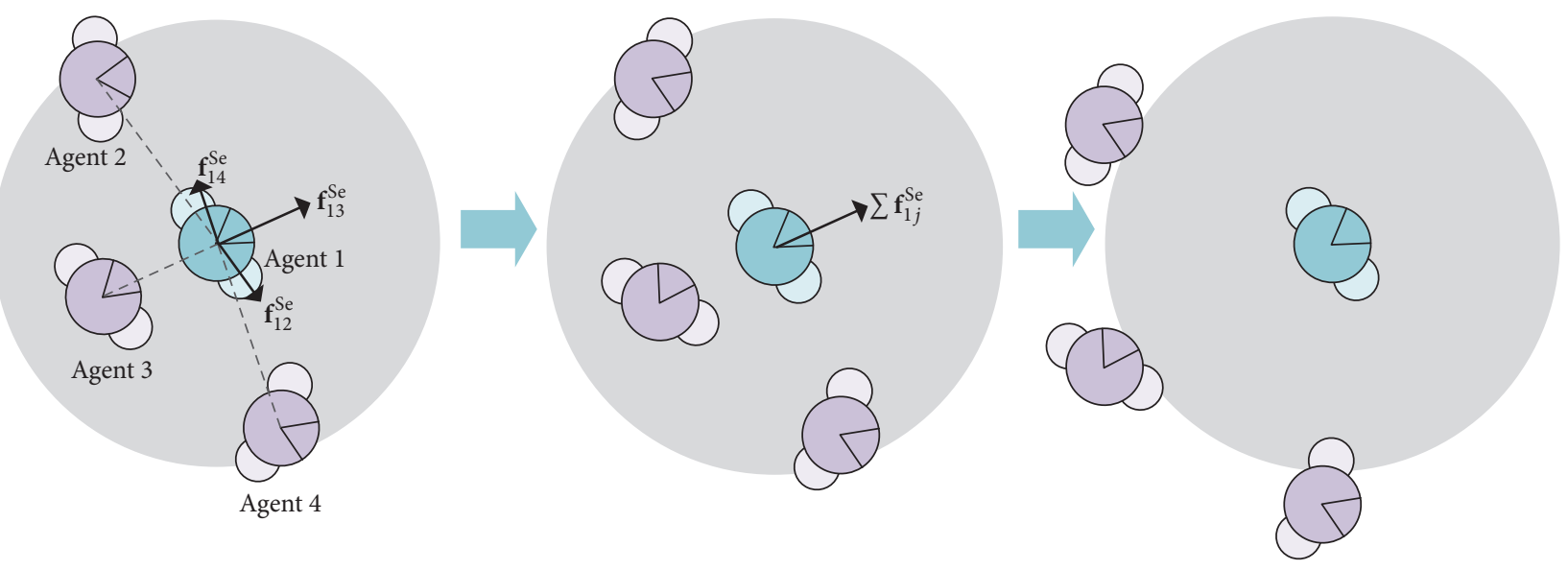

(a)
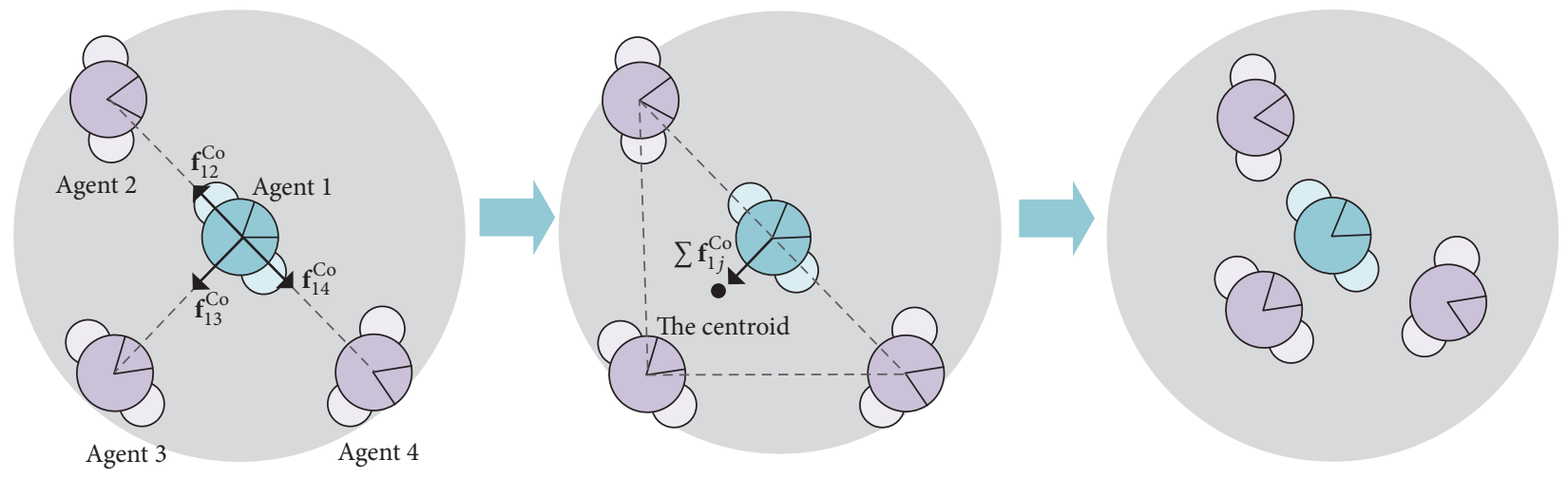

(b)
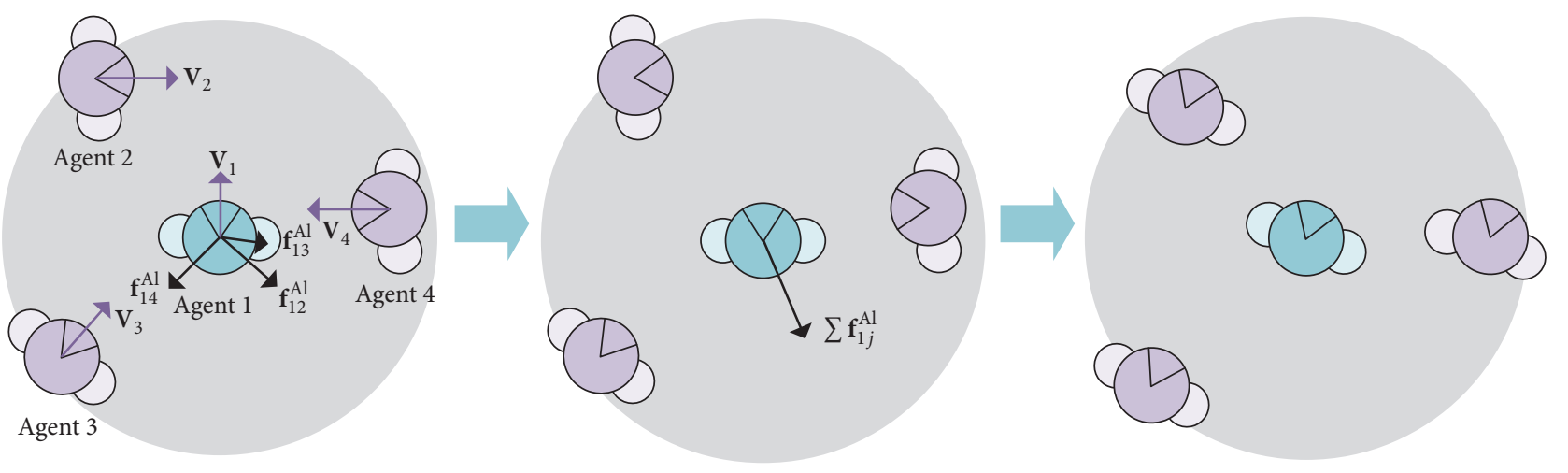

(c)

FIGURE 3: The steering forces of (a) separation, (b) cohesion, and (c) alignment.

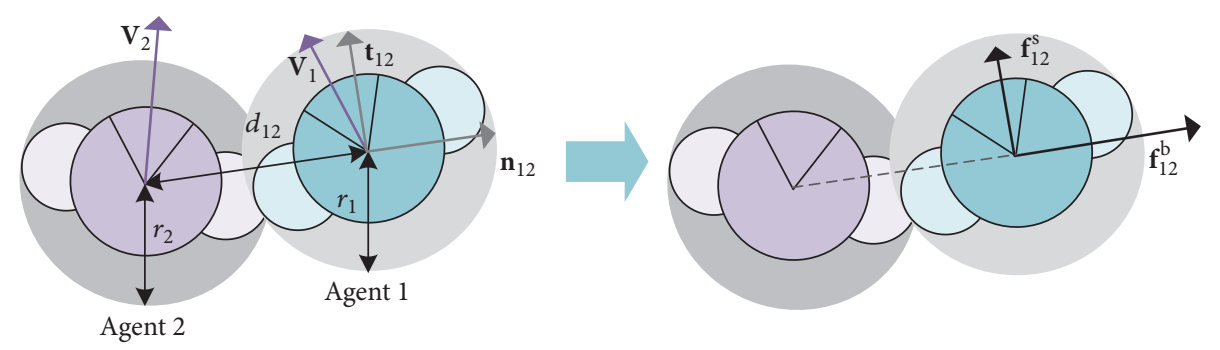

FIGURE 4: The body force and the sliding friction force. 


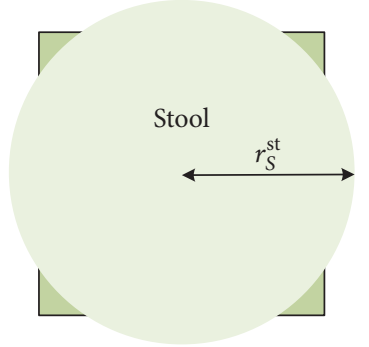

(a)

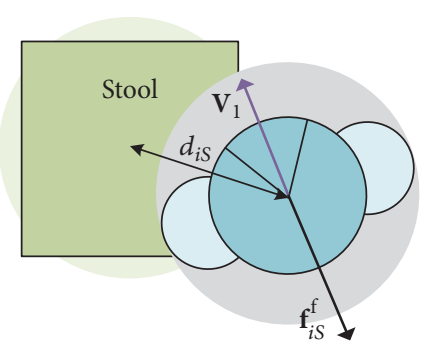

(b)

FIGURE 5: The approximate radius of the stool and the resistance force from the stool.

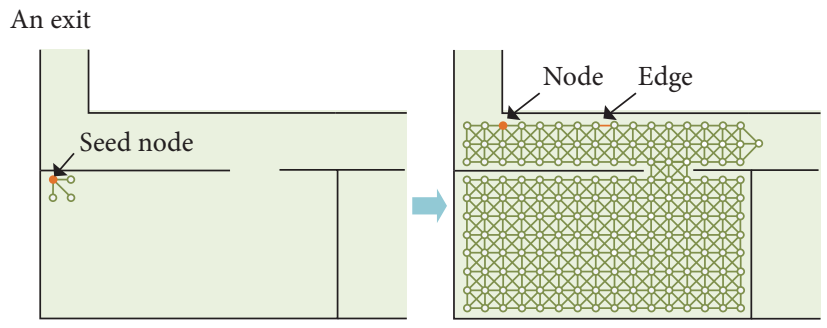

(a)
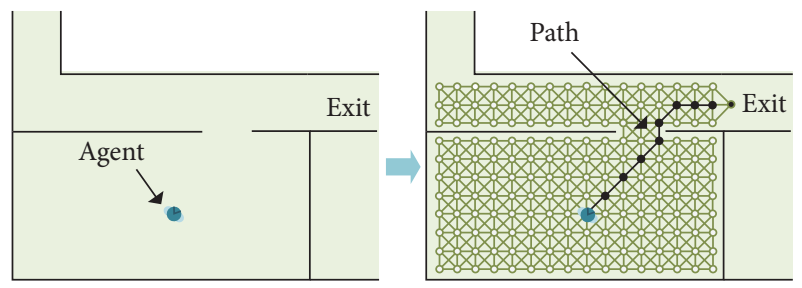

(b)

FIGURE 6: An example of a path planning graph. (a) The generation of the graph and (b) the shortest path on the graph calculated by Dijkstra.
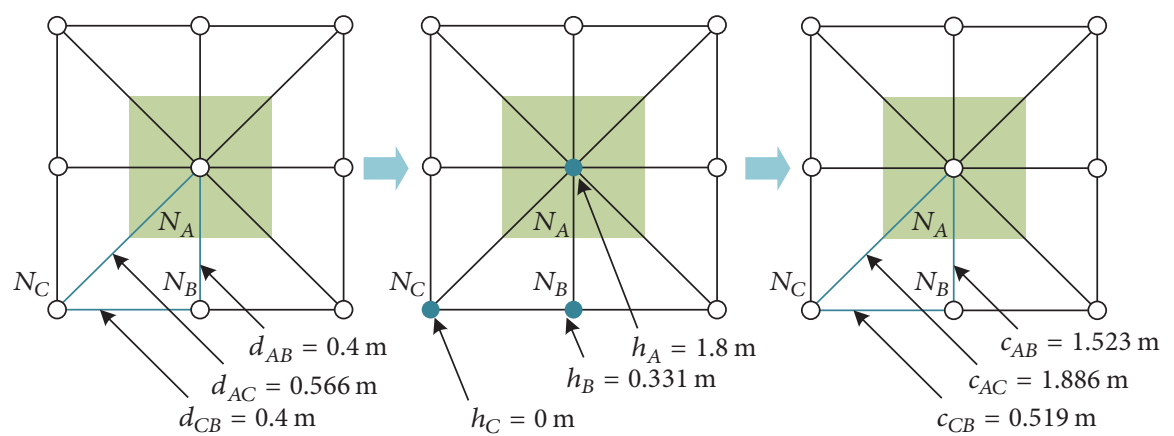

FIGURE 7: An example of the calculation of the cost of the edges near a stool.

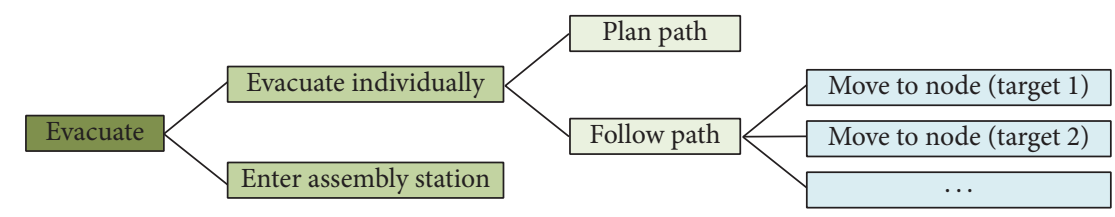

(a)

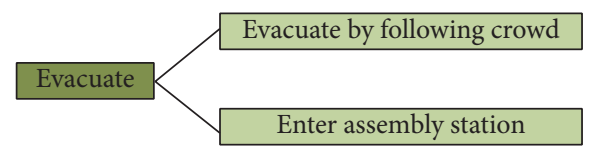

(b)

Figure 8: Process of decomposing and satisfying goals.

of the evacuation, an individual agent desires to evacuate and thus pursues the evacuate goal. This goal is a composite goal and cannot be executed unless it is decomposed into subgoals. As shown in Figure 8(a), the evacuate goal can be broken down into evacuate individually and enter assembly station goals and the evacuate individually goal can be further decomposed into the plan path and follow path goals. The plan path goal is satisfied by requesting the path planning model to plan a path to the assembly station, after which it is deleted from the goal list. The agent then pursues the follow path goal that can be decomposed into a series of prime move to node goals. Each of the move to node goals contains a target node on the shortest path as calculated by the path planning model. The position of the target node is the $\mathbf{p}_{i}^{0}(t)$ needed in (4). When the agent is surrounded by others it will delete the evacuate individually goal and its subgoals and add the evacuate by following the crowd goal to the goal list (see Figure 8(b)). The process of decomposing 


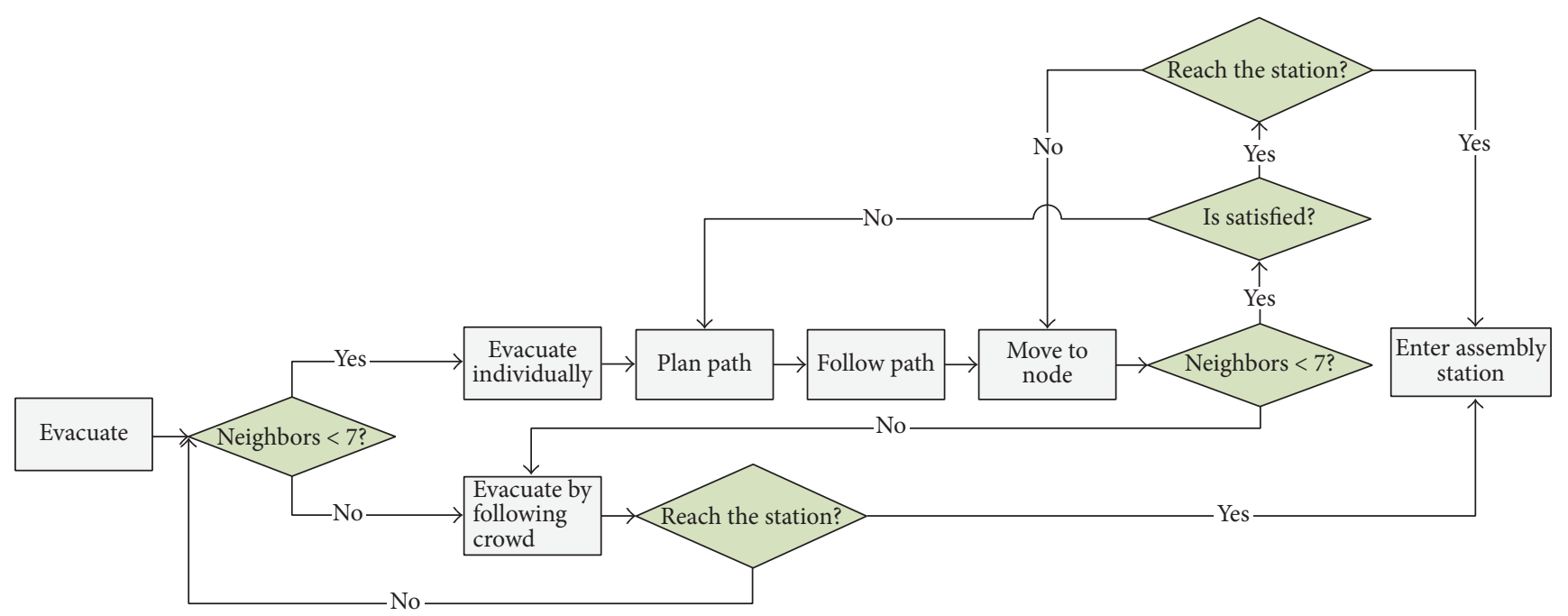

FIGURE 9: A flow diagram illustrating the process of an agent pursuing goals.

and satisfying goals continues until all the goals have been executed.

Figure 9 presents a flow diagram of an agent pursuing certain goals involved in this model and their associated responses. The rectangles denote the different goals, the rhombuses represent the judgment conditions, and the arrows indicate the direction of action flow. The number of neighbors surrounding an agent determines whether he/she evacuates individually or follows the crowd. If the number of neighbors is less than 7, the evacuate individually goal is added to the goal list; otherwise, the evacuate by following crowd goal is added. While pursuing the evacuate individually goal an agent does not consider the separation force $\mathbf{f}_{i j}^{\text {se }}$ and the cohesion force $\mathbf{f}_{i j}^{\mathrm{co}}\left(\mathbf{f}_{i j}^{\mathrm{se}}=0\right.$ and $\left.\mathbf{f}_{i j}^{\mathrm{co}}=0\right)$. Note that sometimes the agent encounters congestion and cannot move to the node in time, in which case the move to node goal is not satisfied and the agent needs to plan a potentially different path to the assembly station. The agent will pursue the enter assembly station goal if this goal is satisfied and the agent can reach the assembly station; otherwise, it will execute another move to node goal with the next node in the path.

\section{Parameter Selection}

All the simulations in this paper were run on a PC with an Intel Core (TM) i5 1.7 GHz CPU and 4 GB of memory. And the model was developed using $\mathrm{C}++$ in a Microsoft Visual 2013 environment. Table 1 lists the parameters of the agent movement model. Some of the parameters are relevant to the physical traits and demographics of the passengers, such as $m$, $v^{\max }$, and $r$, whereas others are related to the nature of the movement model itself. $k_{\mathrm{f}}$ is estimated from simulations and others are taken from the literature [1, 3, 14, 31]. A default value of parameter (except $v^{\max }, r$ ) was used for all passengers in order to minimise parameter number for the sake of robustness and adjustment while acknowledging that in reality parameter values differ somewhat among

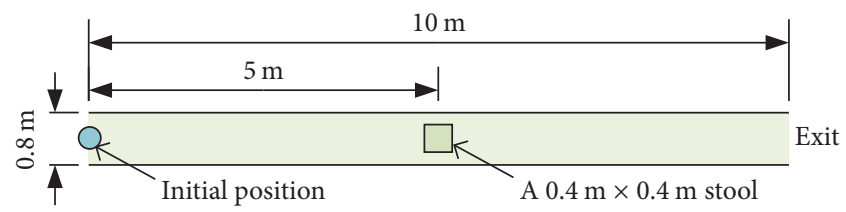

FIGURE 10: Test geometries used for parameter selection of $k_{\mathrm{f}}$.

passengers. It is worth noting that the default value of $k_{\text {se }}$ is drawn from [14] while $k_{\mathrm{se}}, k_{\mathrm{co}}$, and $k_{\mathrm{al}}$ have their relative proportion $(10: 2: 4)$ taken from [31]. Then, we get the initial values of $k_{\text {co }}$ and $k_{\text {al }}$ (see Table 1 ).

We set the corridor size to $10 \mathrm{~m} \times 0.8 \mathrm{~m}$ and analyzed the effect of parameter $k_{\mathrm{f}}$ on the evacuation time. In these simulations, each passenger walked across the corridor at $1.0 \mathrm{~m} / \mathrm{s}$ from the left-hand side to the right. Ten simulations were run for each value of $k_{\mathrm{f}}$. Figure 10 shows $k_{\mathrm{f}}$ against evacuation time. We selected $k_{\mathrm{f}}=1500 \mathrm{~kg} \mathrm{~s}^{-1}$ as the default value so that it took a passenger about $2 \mathrm{~s}$ to traverse the stool in addition to the $10 \mathrm{~s}$ needed to cross the corridor when no stool was present (Figure 11).

\section{Model Validation}

4.1. Comparison with a Floor Field Model. Figure 12 shows models of two typical underground shopping streets. One hundred pedestrians walking at $0.8 \mathrm{~m} / \mathrm{s}$ were set as the initial condition. Ten simulations were run for each arrangement. Table 2 compares the evacuation times found in this study with those from a previous paper [45], which showed a good match. The evacuation times predicted by our model come with a larger standard deviation, in agreement with the randomness of evacuation events. Moreover, our model is more efficient in calculation, taking less time.

We investigated the computational costs of the proposed model, by running evacuation simulations of different population in the aforementioned two typical underground 
TABLE 1: Default values used in crowd-movement model.

\begin{tabular}{lcccc}
\hline Parameter & Default value & Unit & Description & Source \\
\hline$m$ & 80 & $\mathrm{~kg}$ & Passenger mass & Literature [14] \\
$v^{\max }$ & $0.97-1.62$ & $\mathrm{~m} \mathrm{~s}^{-1}$ & Maximum passenger walking speed & Literature [1] \\
$r$ & $0.25-0.29$ & $\mathrm{~m}$ & Passenger radius & Literature [3] \\
$\tau$ & 0.5 & $\mathrm{~s}$ & Characteristic time & Literature [14] \\
$k_{\mathrm{r}}$ & 2000 & $\mathrm{~N}$ & Strength of repulsive interaction force & Literature [14] \\
$k_{\mathrm{b}}$ & 120,000 & $\mathrm{~kg} \mathrm{~s}^{-2}$ & Strength of body force & Literature [14] \\
$k_{\mathrm{s}}$ & 240,000 & $\mathrm{Kg} \mathrm{m}^{-1} \mathrm{~s}^{-1}$ & Strength of sliding friction force & Literature [14] \\
$R$ & 1.0 & Dimengion & Neighborhood radius & Literature [31] \\
$B$ & 0.08 & $\mathrm{~kg} \mathrm{~m}^{2} \mathrm{~s}^{-2}$ & Growth factor of compression force & Literature [31] \\
$k_{\mathrm{se}}$ & 2000 & $\mathrm{~kg} \mathrm{~s}^{-2}$ & Strength of separation force & Literature [14] \\
$k_{\mathrm{co}}$ & 400 & $\mathrm{~kg} \mathrm{~s}^{-1}$ & Strength of cohesion force & Literature [14, 31] \\
$k_{\mathrm{al}}$ & 800 & Strength of alignment force & Literature [14, 31] \\
$k_{\mathrm{f}}$ & 1500 & $\mathrm{~kg} \mathrm{~s}^{-1}$ & Strength of friction force from stools & Simulation \\
\hline
\end{tabular}

TABLE 2: Comparison of simulation results from the proposed model and a floor field model.

\begin{tabular}{lccccc}
\hline & \multicolumn{2}{c}{ Spatial model 1 } & \multicolumn{2}{c}{ Spatial model 2 } & Average calculation time (s) \\
& Mean (s) & Standard deviation (s) & Mean (s) & Standard deviation (s) & 31.7 \\
\hline Floor field model [45] & 125.5 & 0.33 & 145.3 & 1.18 & 3.04 \\
The proposed model & 120.4 & 3.14 & 139.8 & 6.11 & 3.04 \\
\hline
\end{tabular}

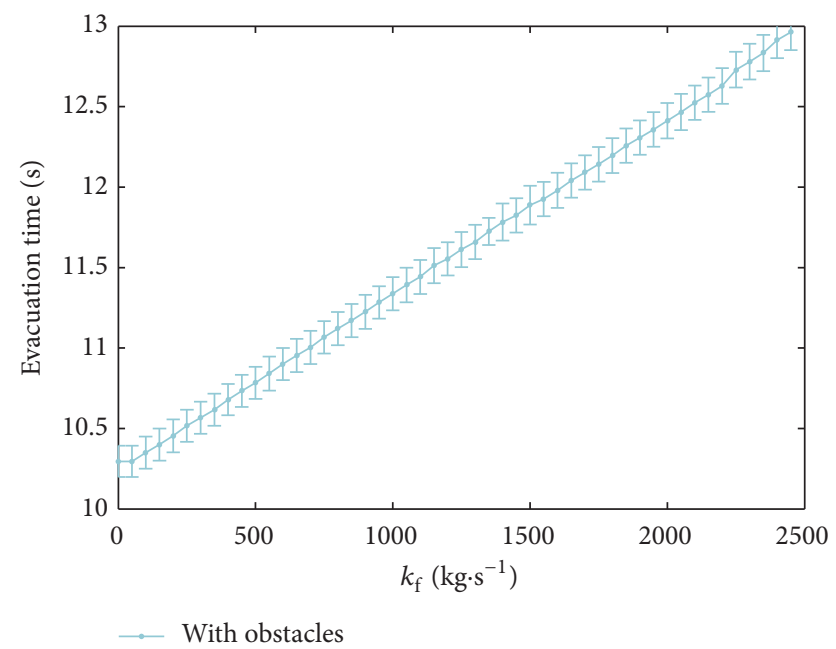

FIGURE 11: The relationship between $k_{\mathrm{f}}$ and evacuation time.

shopping streets. The calculation results are shown in Figure 13, from which it can be seen that the two sets of data show the same trend, indicating that the computational cost of the model we proposed increases linearly with the number of pedestrians. For small scenes, the model we proposed computes faster than real-time calculations. In addition, so far we have not adopted the parallel computation to speed up the calculation, but we will consider it in the future work.

\subsection{Comparison with a Cellular Automaton Simulation of a} Restaurant. We compared our model with a cellular automaton simulation [37] of a restaurant $11.2 \mathrm{~m} \times 7.2 \mathrm{~m}$ in size, with 110 persons and 18 tables (Figure 14). The initial positions of the persons and the walking speed of $1 \mathrm{~m} / \mathrm{s}$ were taken from previous studies $[37,38]$. We analyzed the effect of varying the width of an exit door in the center of the left-hand wall, both with and without the presence of obstacles. As can be seen from Figure 15, the curves matched well. The evacuation time, without presence of obstacles, diminishes as the width of the door grows until a critical width value (being $2.4 \mathrm{~m}$ with our model), beyond which the time no longer diminishes. The evacuation time, with presence of obstacles, decreases as the width of the door grows. Moreover, our model shows more variations when obstacles are present.

4.3. Flows in Corridors. The empirical relation between flow and density can be represented by a fundamental diagram. It is widely used in handbooks and the design of pedestrian facilities. Furthermore, it is a quantitative benchmark for models of pedestrian dynamics [48]. We used the geometry shown in Figure 16 to compare our simulation results using different numbers $(50,100,150,200,250,300,350$, and 400$)$ of pedestrians. The corridor was modeled as a loop to avoid the effect of inflow and outflow on the boundary conditions. Figure 17 compares the simulated corridor flows with those from fundamental diagrams. Our predictions agreed well with the fundamental diagrams, showing specific flows first increasing as the crowd density increased and then starting to decrease as the density became sufficiently high to hinder walking. Our predictions for specific human flows were also close to the experimental results of Older [42].

\section{Simulations and Results}

5.1. Case 1: Simulation for Bypassing and Crossing Behavior. In order to demonstrate the effect of bypassing and crossing 


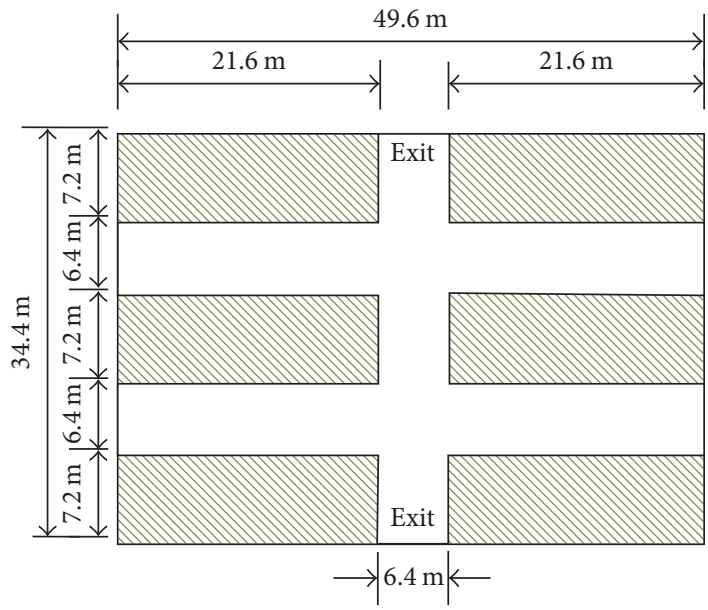

(a)

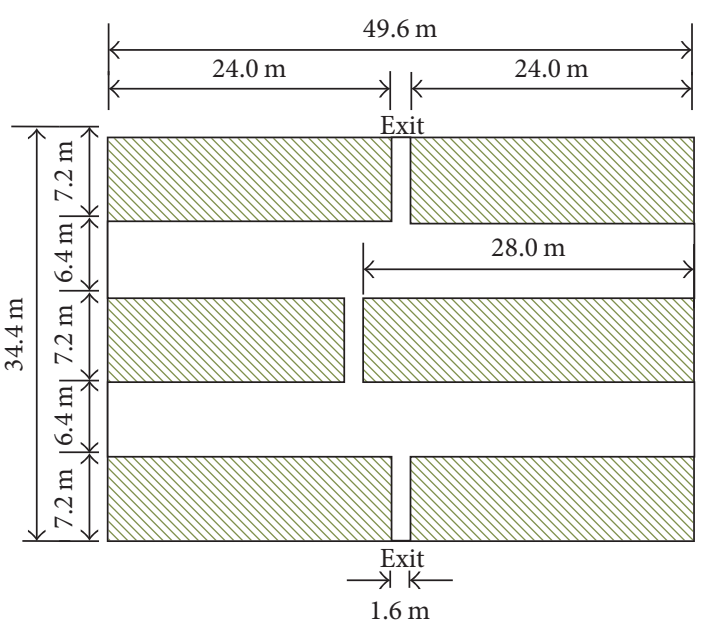

(b)

FIGURE 12: Two typical underground shopping streets. (a) Spatial model 1. (b) Spatial model 2.

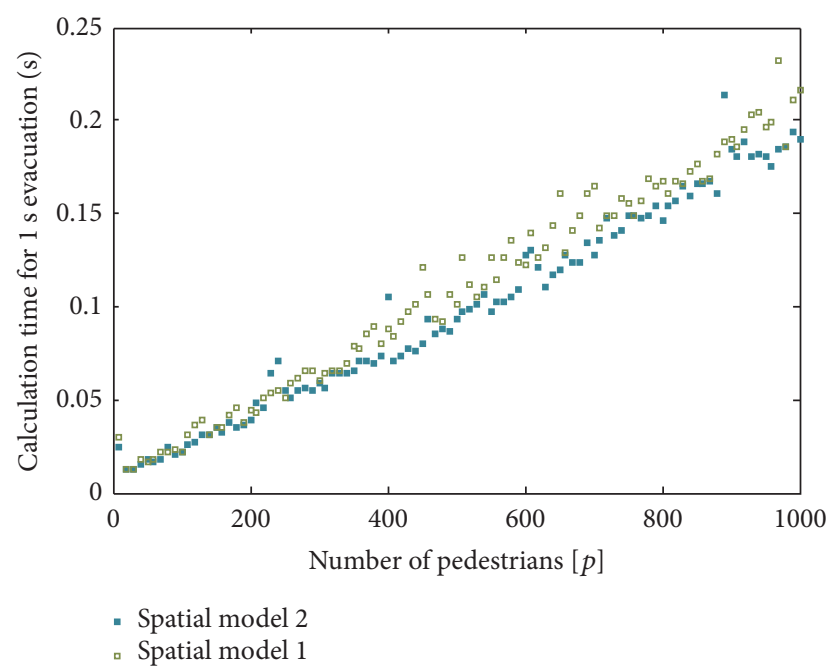

FIGURE 13: Analysis of the computational times of the proposed model.

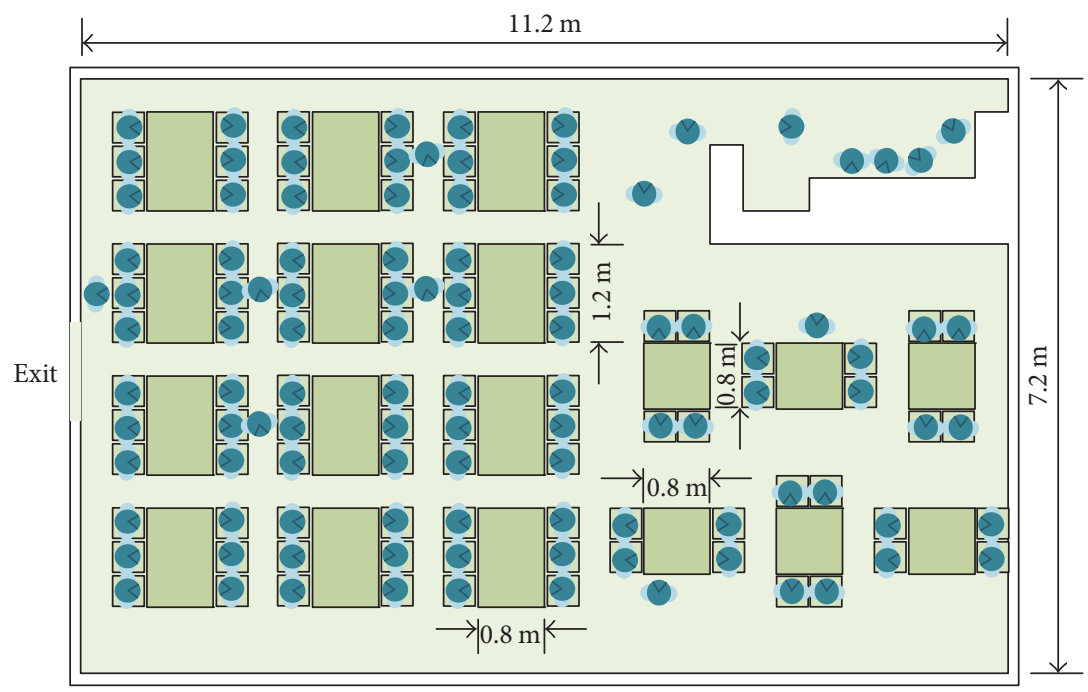

Figure 14: A restaurant with 18 tables and 110 persons. 


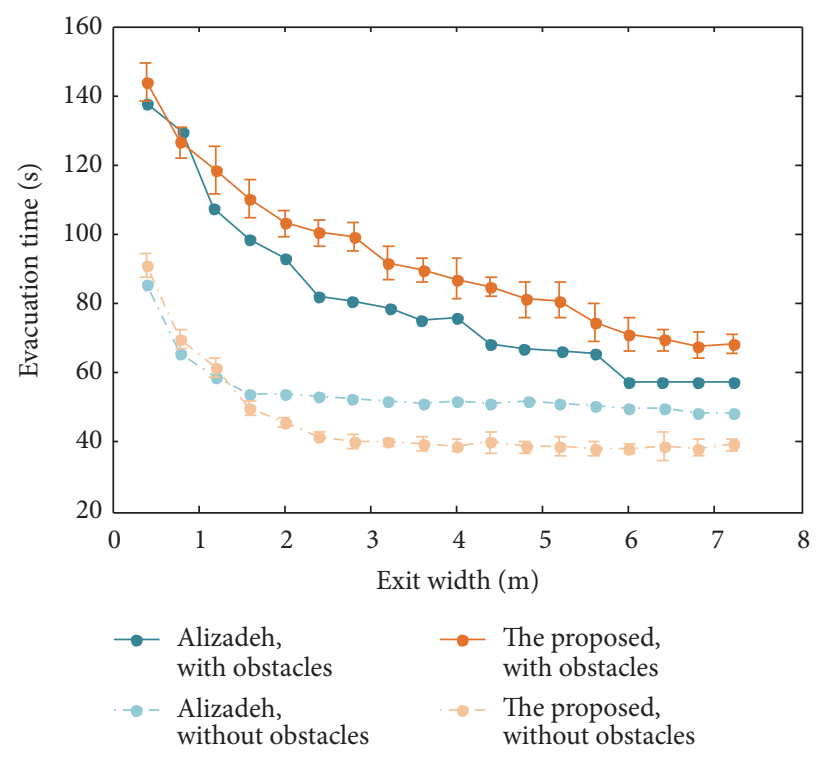

Figure 15: Evacuation time against the exit door width.

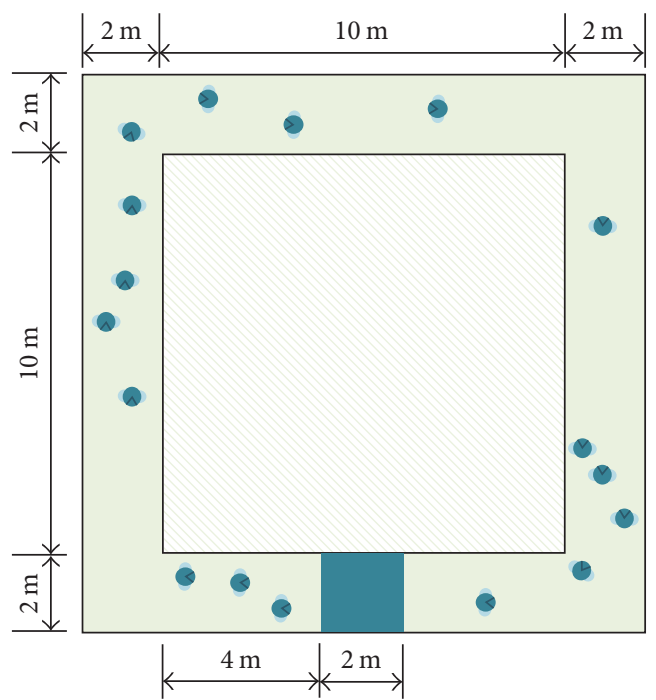

FIGURE 16: Corridor arrangement used for calculating specific flows; the blue rectangles indicate the regions used to measure the speed-density results.

behaviors, a simulation case with a row of stools and a table with six stools is utilized (see Figure 18). In this case, a passenger is located in a $10 \mathrm{~m} \times 6 \mathrm{~m}$ cabin with a $0.6 \mathrm{~m}$ exit located centrally on the $6 \mathrm{~m}$ wall. Nine $0.4 \mathrm{~m} \times 0.4 \mathrm{~m}$ stools are arranged in a row in the middle of the cabin and are spaced $0.2 \mathrm{~m}$ from each other. A $1.8 \mathrm{~m} \times 0.6 \mathrm{~m}$ table with six corresponding stools is located $2.5 \mathrm{~m}$ from the exit. Assuming a passenger walking to an exit at a speed of $1 \mathrm{~m} / \mathrm{s}$, we simulated the eight scenarios shown in Figure 19.

As can be seen from Figure 19(a), the passenger took $10.05 \mathrm{~s}$ to reach the exit, if there is no stool between the passenger and the exit. Figures 19(b)-19(d) show that when a row of stools was added, the passenger preferred to bypass the stools rather than cross the stools, as this required less time (less than $12.02 \mathrm{~s}$, see Figures 19(e) and 19(f)). As the number of stools was increased, increasing the time required to bypass the stools, the passenger would cross the middle stool (Figures 19(e) and 19(f)). The passenger took a detour, as indicated by the route in Figure $19(\mathrm{~g})$, when a table and its corresponding 6 stools were added.

\subsection{Case 2: Simulation for Evacuation from the Restaurant} Area of a Passenger Ship. We conducted evacuation simulations on a restaurant area in a passenger ship as a case study. The arrangement of Case 2 is schematically illustrated in Figure 20. The size of the restaurant area is approximately $33.4 \mathrm{~m} \times 24.2 \mathrm{~m}$, which includes 9 cabins, 25 doors, 344 stools, 61 tables, 9 food tables, a corridor, and 2 assembly stations. The widths of the doors are $0.8 \mathrm{~m}$ and the width of the corridor is $2.0 \mathrm{~m}$. There are two table sizes: $0.6 \mathrm{~m} \times 1.8 \mathrm{~m}$ (with 

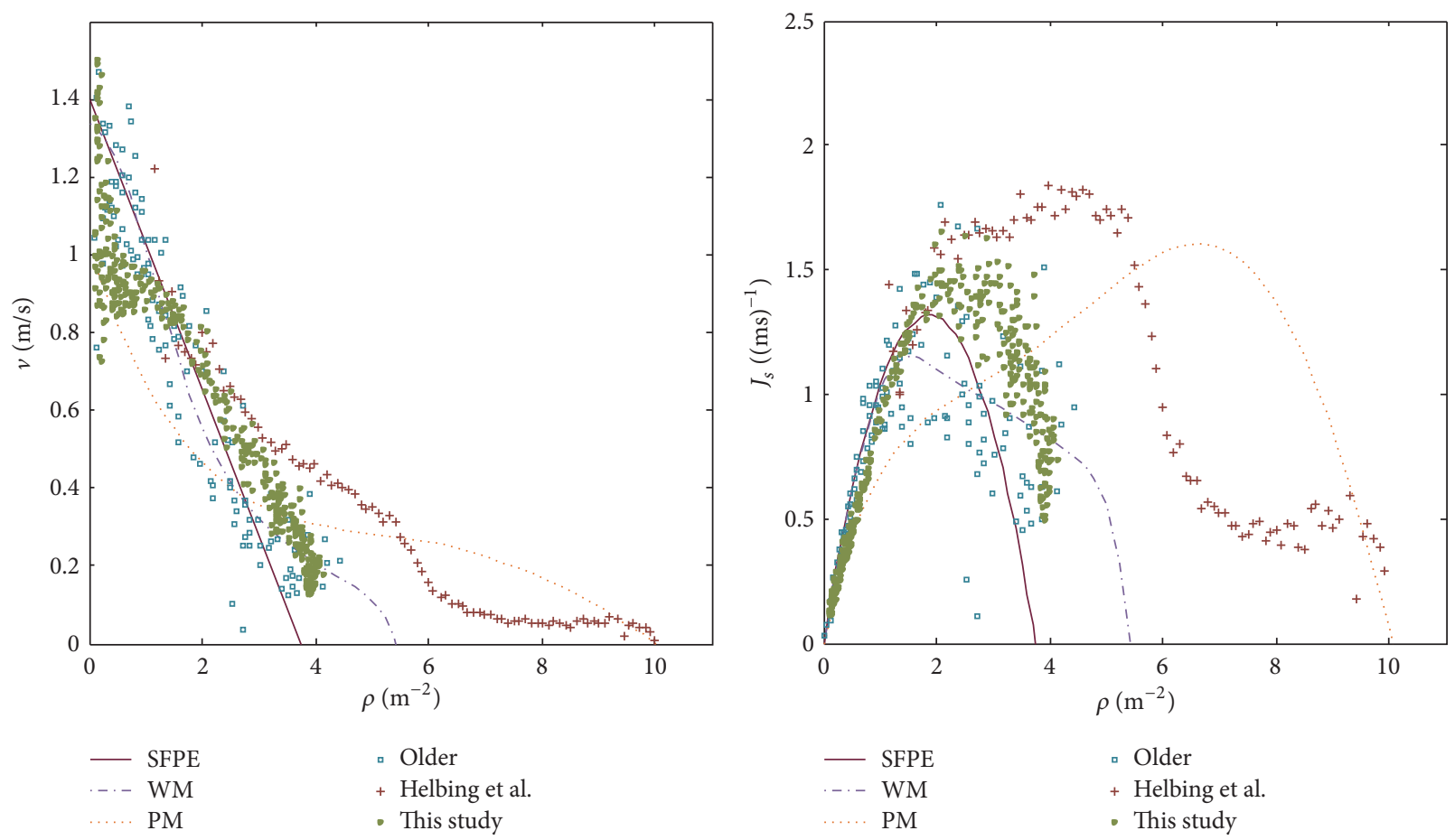

FIGURE 17: Simulation of corridor flows compared with those from fundamental diagrams based on the SPFE Handbook (SPFE) [39], the Predtechenskii and Milinskii (PM) [40] and Hoogendoorn and Daamen [41] Guidelines, and the experimental results of Older [42] and Helbing et al. [43].

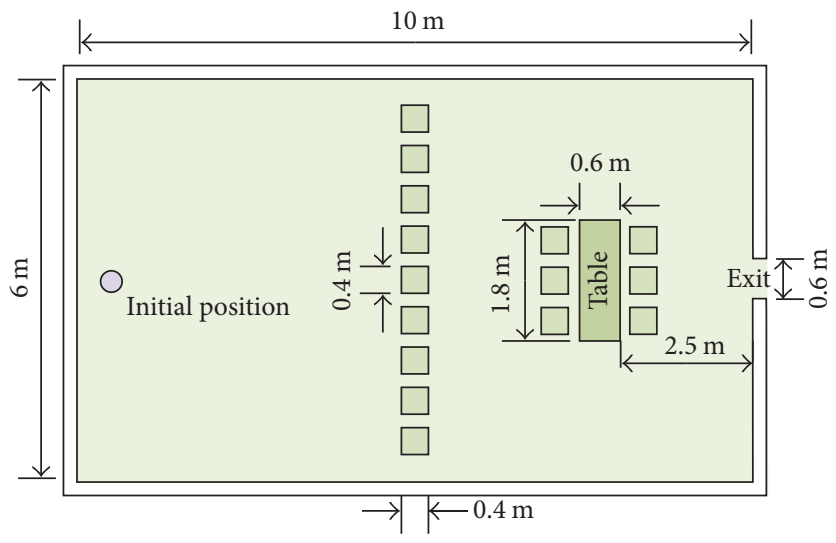

FIgURE 18: The arrangement used in the Case 1 simulation.

6 stools) and $0.6 \mathrm{~m} \times 1.2 \mathrm{~m}$ (with 4 stools). The dimensions of the stools are $0.4 \mathrm{~m} \times 0.4 \mathrm{~m}$. Each cabin contains one $3.6 \mathrm{~m} \times$ $0.6 \mathrm{~m}$ food table. All of the 344 passengers are seated on the stools at the beginning of the evacuation, and their maximum unhindered walking speeds on flat terrain are uniformly distributed over the interval of $0.97-1.62 \mathrm{~m} / \mathrm{s}$ as stipulated in the IMO Guidelines [1]. At the start of the evacuation, the passengers immediately evacuate to their nearest assembly stations. Moreover, we ran a comparative simulation that had the obstacles removed.

Figure 21 presents Case 2 simulation snapshots. We compared simulated passenger distributions for the different arrangements at $t=8 \mathrm{~s}$. The passengers are blocked at the door sooner in the arrangement that included obstacles compared with the arrangement without obstacles. Figure 22 shows the cumulative percentage of evacuated passengers as a function of time for the Case 2 simulation. Surprisingly, it takes less time to evacuate from the arrangement with obstacles compared with the arrangement without obstacles, indicating that the presence of obstacles can speed up the evacuation process.

Density and time are important means of identifying congestion. When the density exceeds a certain critical value (3.5 persons $/ \mathrm{m}^{2}$ ) for a period of time, the occurrence of congestion can be considered [48]. Figure 23 shows the density plots of occupant density exceeding 3.5 persons $/ \mathrm{m}^{2}$. 


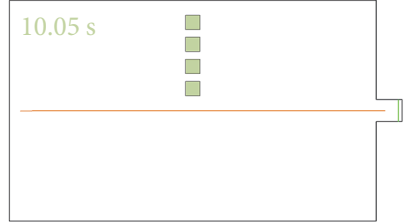

(a)

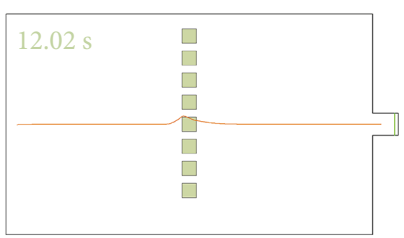

(e)

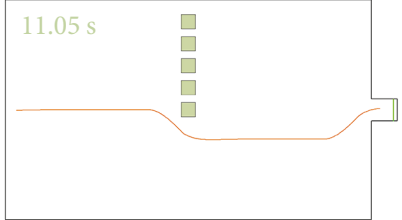

(b)

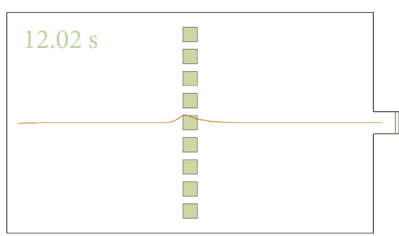

(f)

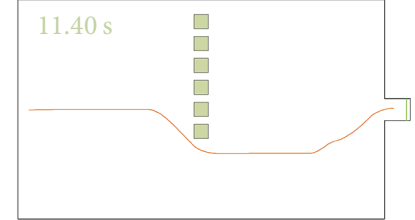

(c)

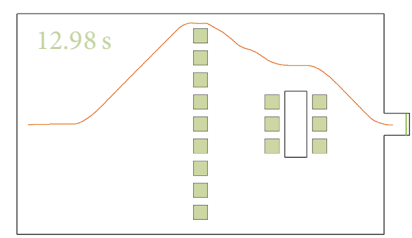

(g)

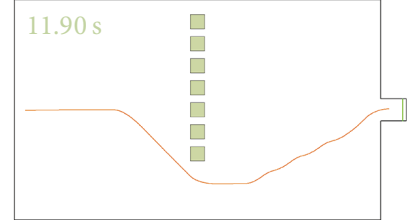

(d)

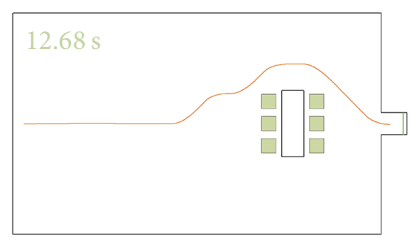

(h)

Figure 19: Simulated passenger routes in Case 1.

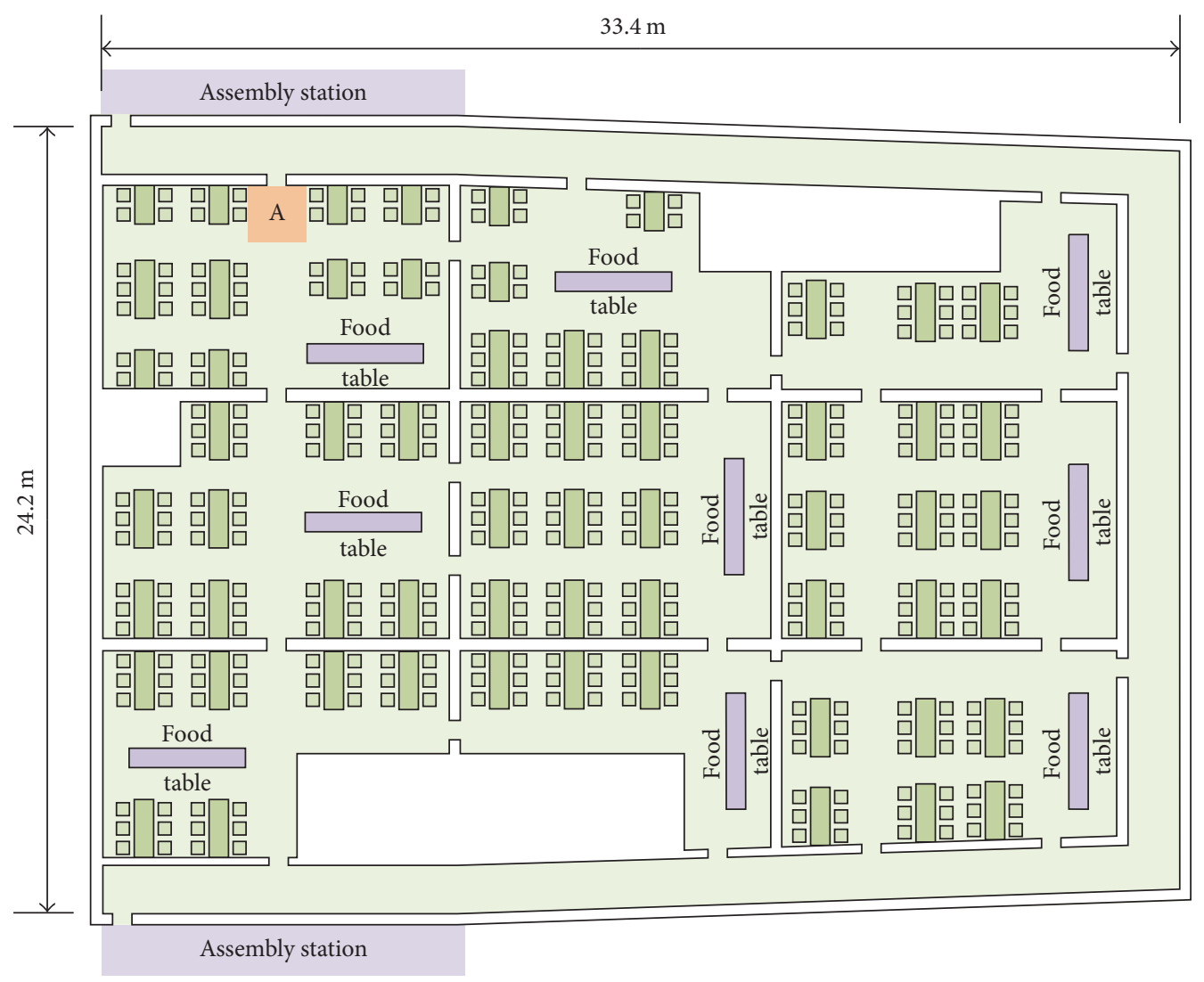

FIGURE 20: The arrangement used in the Case 2 simulation.

It can be seen from Figure 23 that the blocking time in area A (the orange square marked in Figure 20) is longer (the maximum time is $180 \mathrm{~s}$ ) under the scene without stools and tables. That is the reason that it takes longer time to evacuate from the arrangement without obstacles compared with the arrangement with obstacles. It is worth noting that multiple paths can be followed to reach an exit from a cabin, and path length might not be the only reasonable criterion $[29,30]$ (this criterion will reduce the utilization rate of doors far away from the assembly station). Other reasonable criterion will be considered in the future works.

We ran the simulations a total of 2,700 times at different door widths and with different numbers of passengers to evaluate the influence of these parameters on the evacuation time. The results are shown in Figure 24 and they indicate that, for the same number of passengers, the time to evacuate with and without obstacles decreases as the width of door increases and that when the door width is fixed, the evacuation time 

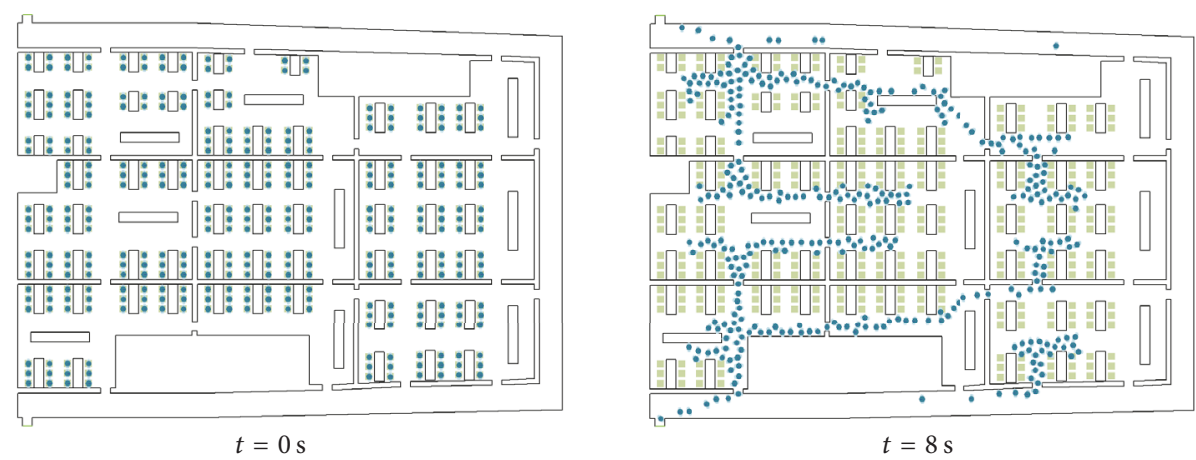

(a)
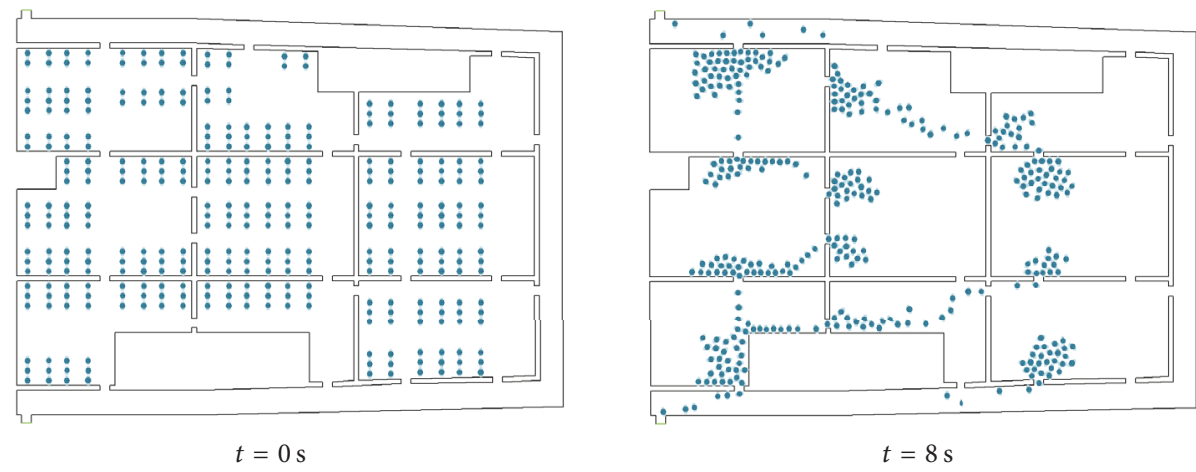

(b)

Figure 21: Snapshots of Case 2 simulation with (a) an arrangement with obstacles and (b) an arrangement without obstacles.

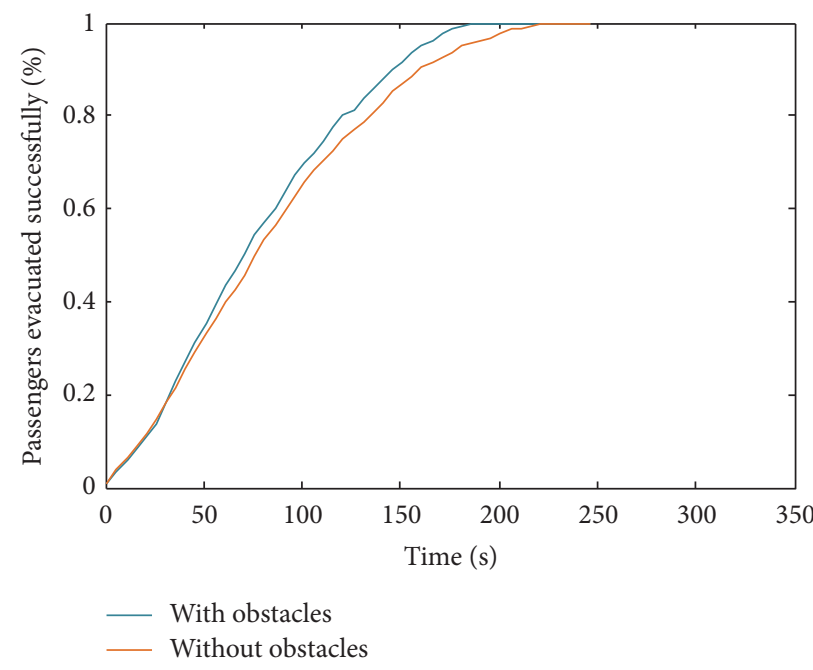

FIGURE 22: Cumulative percentage of evacuated passengers as a function of time for the Case 2 simulation.

increases as the number of passengers increases. These results are in good agreement with previous literature. Comparison of the time to evacuate with and without obstacles leads to the conclusion that the presence of obstacles in Case 2 acts to reduce the evacuation time.

5.3. Case 3: Simulation for Evacuation of a Dining Hall in a Passenger Ship. In order to show the effect of obstacles (stools and tables) on the evacuation time, we ran simulations in a $16.4 \mathrm{~m} \times 15.8 \mathrm{~m}$ dining hall. Four scenarios were designed in Case 3 numbered A-D and are described in the following paragraphs.

Scenario A. 36 tables and 216 stools are arranged in 6 rows and 6 columns (see Figure 25). The sizes of table and chairs are the same as in Case 1. The spaces between different rows are $1 \mathrm{~m}$, and the width between columns is $2 \mathrm{~m}$ (the distance between the middle two columns is $2.8 \mathrm{~m}$ ). A $0.8 \mathrm{~m}$ exit is located at the center of the $16.4 \mathrm{~m}$ wall. There are 216 passengers sitting on the stools at the beginning of the evacuation (see Figure 26(a)). Their maximum unhindered walking speeds on flat terrain are uniformly distributed over the interval of $0.97-1.62 \mathrm{~m} / \mathrm{s}$.

Scenario B. The same as Scenario A except that tables A and B and their corresponding 12 passengers and stools are removed (see Figure 26(b)).

Scenario C. The same as Scenario 2 except that tables C and D and their corresponding 12 passengers and stools are removed (see Figure 26(c)).

Scenario D. The same as Scenario 1 except that most of the tables and stools are removed, with the exception of tables A and B and their corresponding stools (see Figure 26(d)).

In addition, we evaluated the evacuation times for each scenario in contrast to simulations with no obstacles present. We ran 50 simulations for each scenario. 


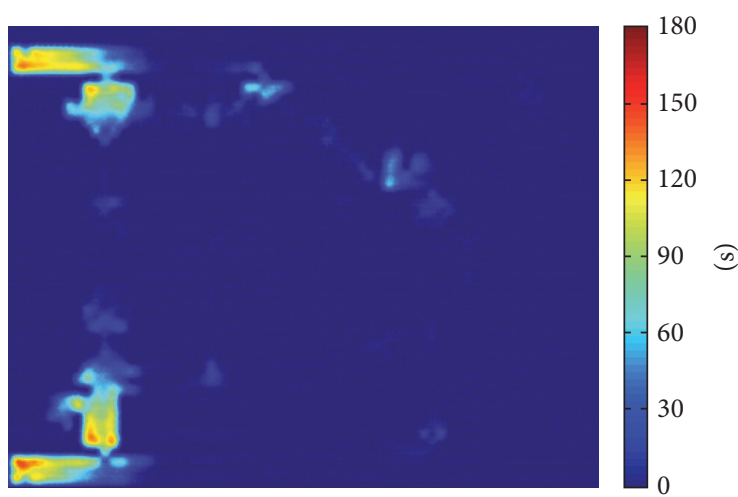

(a)

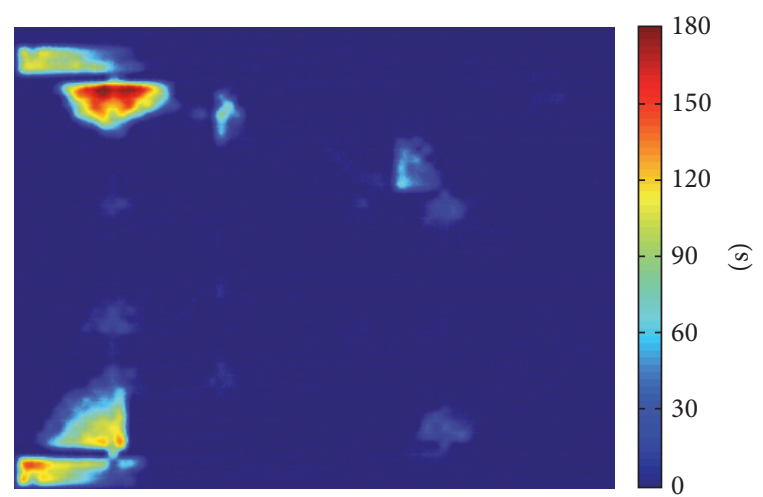

(b)

FIGURE 23: Cumulated density plot of occupant density exceeding the critical value $\left(3.5\right.$ persons $\left./ \mathrm{m}^{2}\right)$; color bar indicating different blocking time. (a) The scene with stools and tables. (b) The scene without stools and tables.

TABLE 3: The evacuation times and time ratios between different arrangements in Case 3.

\begin{tabular}{lcccc}
\hline & Scenario A & Scenario B & Scenario C & Scenario D \\
\hline Number of passengers $(p)$ & 216 & 204 & 192 & 204 \\
Mean evacuation time with obstacles $T_{1}(\mathrm{~s})$ & 295.7 & 333.7 & 301.1 & 295.0 \\
Mean evacuation time without obstacles $T_{2}(\mathrm{~s})$ & 315.2 & 311.9 & 298.7 & 315.2 \\
Standard deviation of $T_{1}(\mathrm{~s})$ & 16.0 & 22.3 & 16.7 & 9.4 \\
Standard deviation of $T_{2}(\mathrm{~s})$ & 14.6 & 15.7 & 18.8 & 14.6 \\
Ratio of the evacuation times $T_{1} / T_{2}$ & 0.94 & 1.07 & 1.01 \\
\hline
\end{tabular}

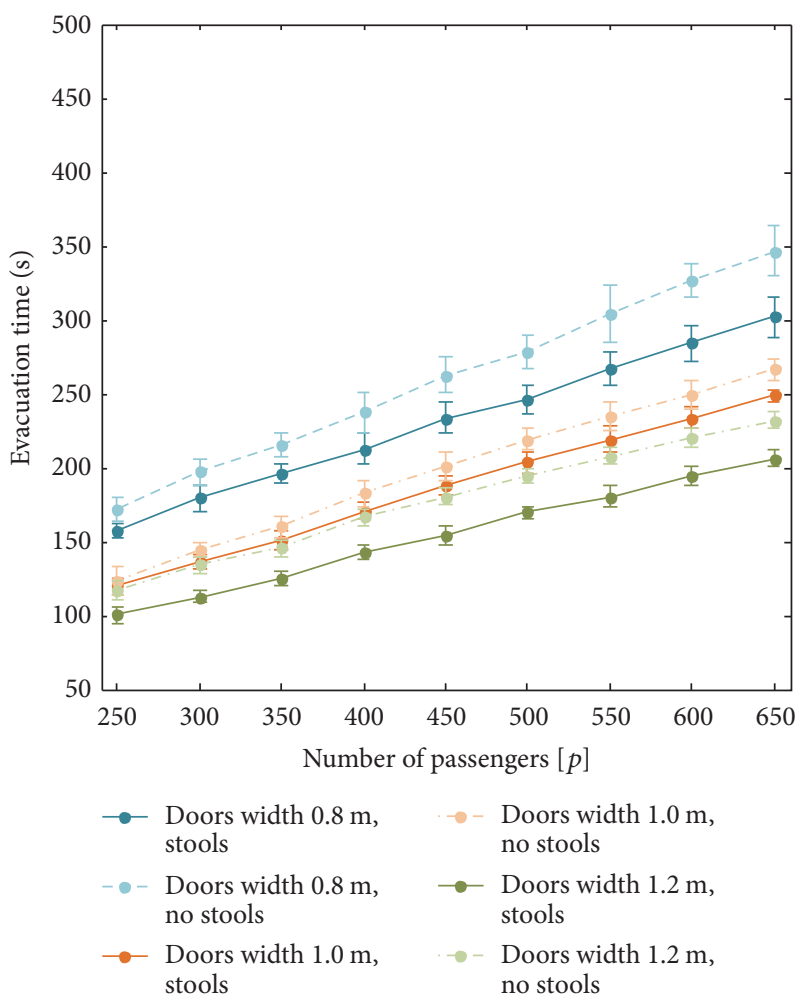

FIGURE 24: Mean evacuation times and standard deviations at different door widths and passenger numbers in Case 2.

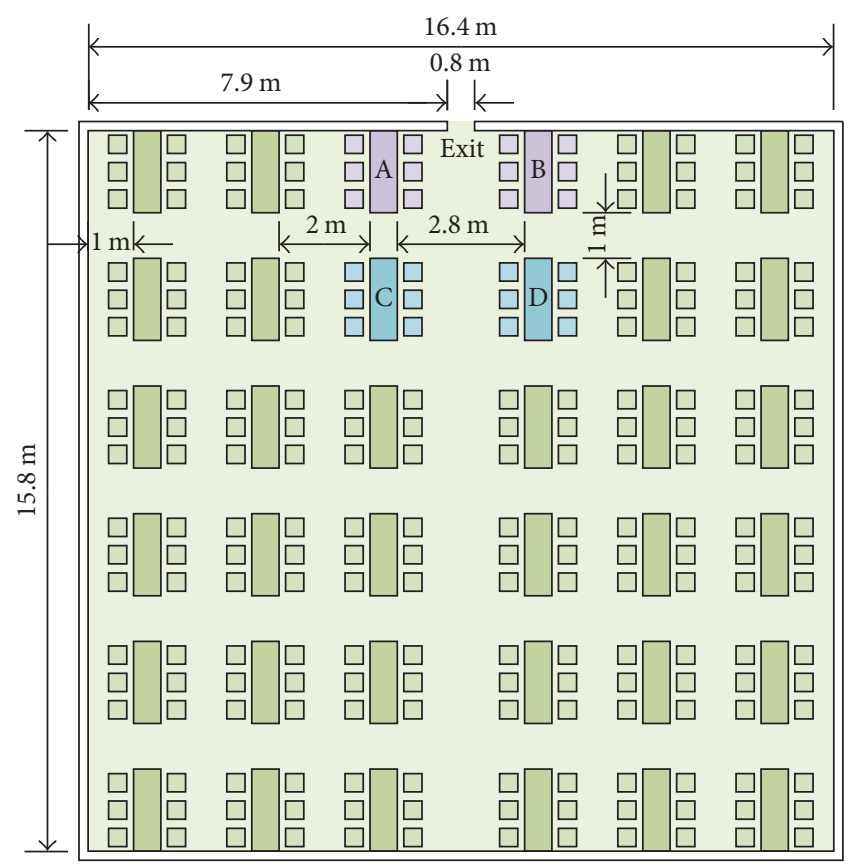

Figure 25: The arrangement used in the Case 3 simulation.

The evacuation times and the time ratios between the different arrangements of each scenario are listed in Table 3. In Scenario $B, T_{1}$ for the default arrangement with obstacles 


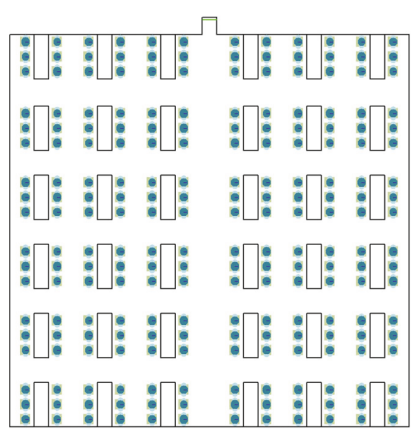

(a)

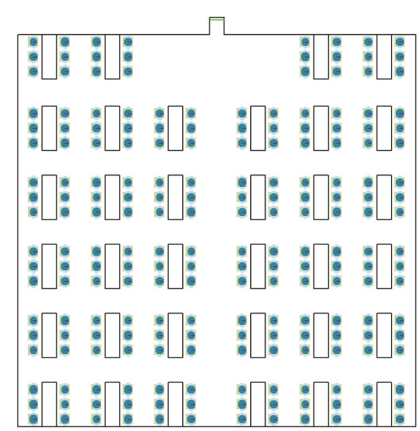

(b)

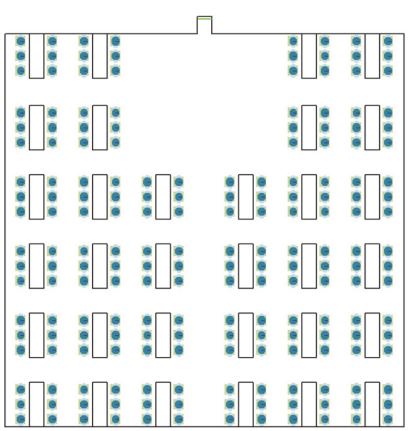

(c)

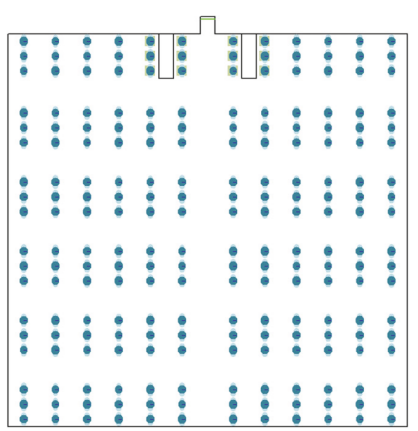

(d)

Figure 26: Passenger distributions in Case 3 simulations of the different scenarios: (a) Scenario A, (b) Scenario B, (c) Scenario C, and (d) Scenario D.

(stools and tables) is $333.7 \mathrm{~s}$ and is greater than the $T_{2}$ (311.9 s) for the arrangement without obstacles. The ratio between $T_{1}$ and $T_{2}$ is 1.07, which indicates that evacuation time increases by about $7 \%(21.8 \mathrm{~s})$ if obstacles are present. In Scenario C the evacuation efficiency is almost the same whether or not obstacles are present. However, in Scenario A the evacuation time drops by approximately $6 \%$ with obstacles present. Therefore, we conclude that the presence of tables $\mathrm{A}$ and $B$ and their corresponding stools increases the evacuation efficiency of the arrangement with obstacles. This conclusion can be also obtained by the comparison of $T_{1}$ and $T_{2}$ in Scenario D.

Figure 27 presents space utilization diagram in the Case 3 simulation. In Scenario $\mathrm{A}$ and Scenario $\mathrm{D}$, the presence of tables $\mathrm{A}$ and $\mathrm{B}$ alters the shape of the clogged passengers (see Figures 27(a) and 27(d)) and increased the evacuation efficiency in comparison with Scenario B. These results can be understood if we consider that tables $\mathrm{A}$ and $\mathrm{B}$ and their corresponding stools restrict the movement of the passengers in the directions perpendicular to the outlet and limit the possible directions in which passengers can scramble towards the exit. This restriction of the passengers' movement acts to mitigate pushing near the exit, which otherwise causes additional friction effects and reduces evacuation efficiency. Furthermore, the shape enclosed by tables A and B, their corresponding stools, and the walls near the exit resemble a funnel to some extent. In order to further demonstrate that changing the clogging passengers' movement direction can affect evacuation efficiency, we ran a series of simulations using Case 4, which is discussed in the following section.

5.4. Case 4: Simulation for Changing the Movement Direction of Clogging Passengers. We ran the simulations of Case 4 in a $20 \mathrm{~m} \times 10 \mathrm{~m}$ cabin with different angles $\Phi$ (see Figure 28 ). A $0.8 \mathrm{~m}$ exit is located at the center of the $10 \mathrm{~m}$ wall. All the passengers, aged 30-50, are uniformly distributed in a $7 \mathrm{~m}$ $\times 10 \mathrm{~m}$ area at the far right of the cabin. Their maximum unhindered walking speeds on flat terrain are uniformly distributed over the interval of $0.97-1.62 \mathrm{~m} / \mathrm{s}$ [1].

Figure 29 presents evacuation times as a function of angle $\Phi$ for 200, 250, and 300 passengers. The results indicate that funnel-shaped exits at the bottleneck act to speed up evacuation. For the same angle, the more the passengers present, the longer the evacuation time that is needed. The trend of the curves indicates that evacuation time increases with the increasing angle $\Phi$. In Figure 30 the results for outgoing flows through exits calculated by the proposed model are compared to some other simulation programs and experimental result of Seyfried [49]. The simulation results of the programs FDS + Evac, MASSEgress, and Simulex are extracted from [50]. The results show that we have a good match with FDS + Evac and MASSEgress software but differ a lot from the results of the software Simulex and Seyfried. The different arrangements of the experiment in many details (e.g., the geometry of the room, the initial density of the pedestrians, the walking speed of the pedestrians, and crowdedness) may contribute to the differences in the calculation results. Figure 31 presents space utilization diagram in the Case 4 simulation. The shape of the clogging passengers changes as the angle changes.

Figure 32 shows the density plots of occupant density exceeding 3.5 persons $/ \mathrm{m}^{2}$. When the density exceeds a certain critical value $\left(3.5\right.$ persons $\left./ \mathrm{m}^{2}\right)$ for a period of time, the occurrence of congestion can be considered. It can be seen from Figure 32 that the blocking time increases with the increasing angle $\Phi$ (the larger the red area, the longer the congestion time). When the angle is small, fewer directions of passengers scramble towards the exit and the passengers quickly pass through the exit. As the angle increases, the passengers scramble in many directions towards the exit and they are blocked at the exit (the passengers form an arch shape around the exit). Overall, obstacles near an exit that reduces the blocked passengers' movement directions act to increase evacuation efficiency.

\section{Conclusion}

Previous studies of the evacuation of passenger ships neglect to include the interior layout of the cabins and treated the cabins as empty rooms. This study investigated the influence of the tables and stools in the cabins of a passenger ship using a new agent-based social force evacuation model. In the current model, each table is treated as an area enclosed with 


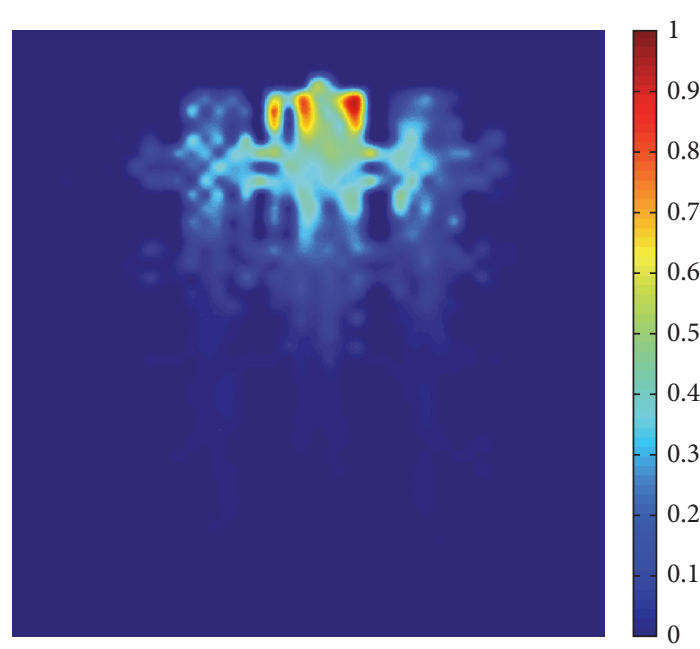

(a)

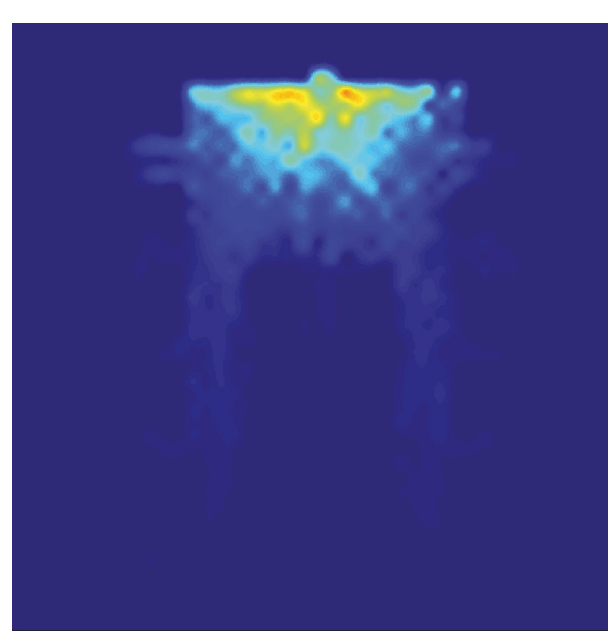

(c)

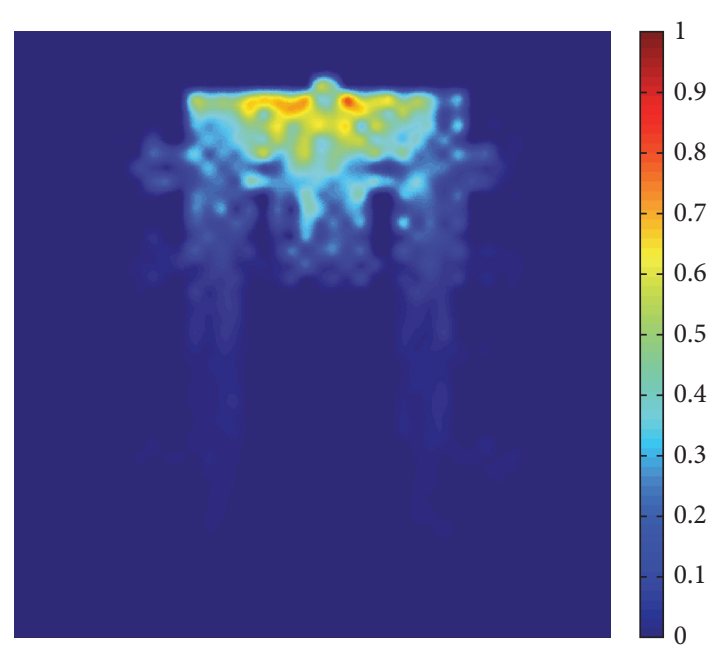

(b)
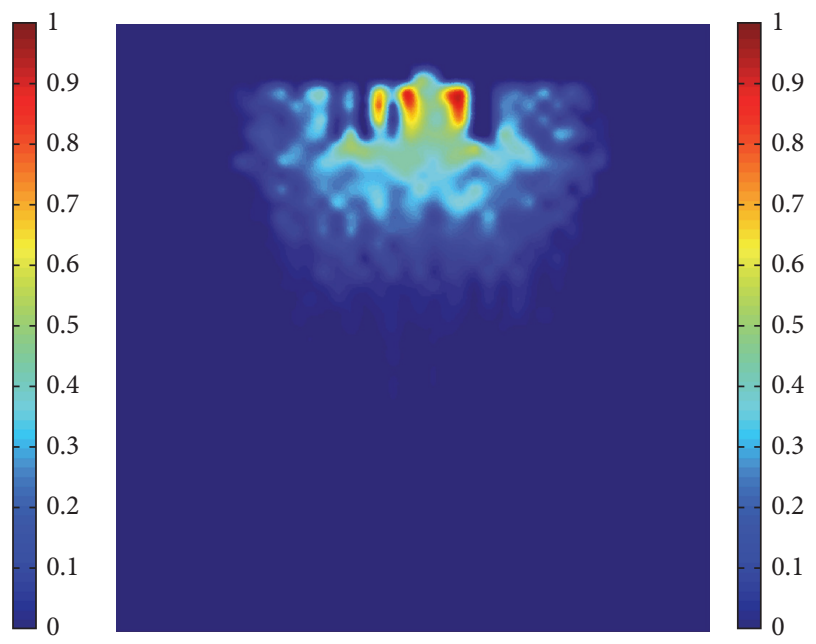

(d)

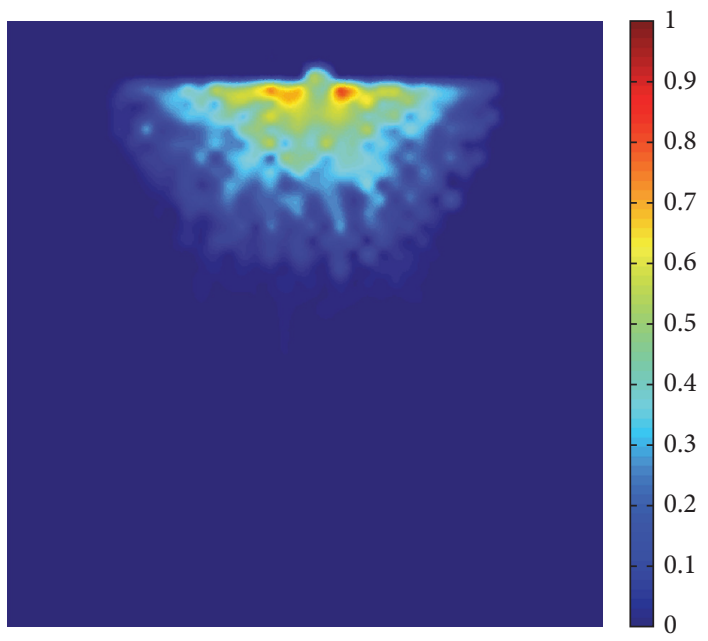

(e)

FIgURE 27: Space utilization diagram for Case 3; color bar indicating different occupied frequencies. (a) Scenario A, with stools and tables, (b) Scenario B, with stools and tables, (c) Scenario C, with stools and tables, (d) Scenario D, with stools and tables, and (e) Scenario A, without stools and tables. 


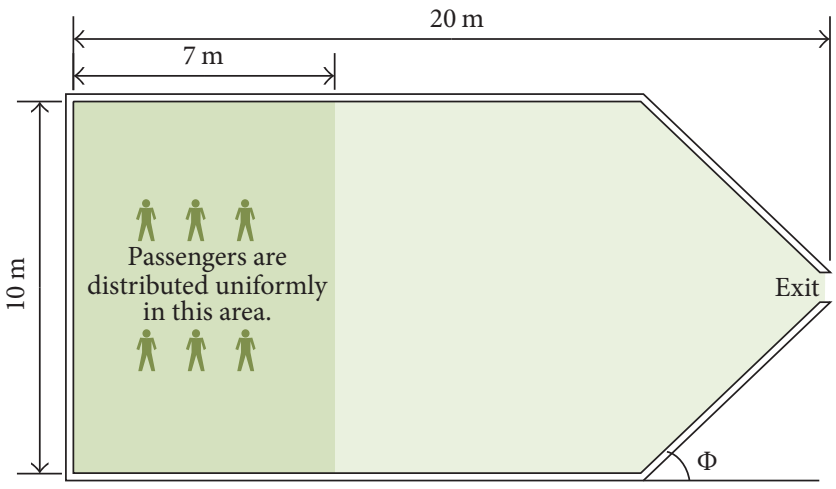

Figure 28: The arrangement used in the Case 4 simulations.

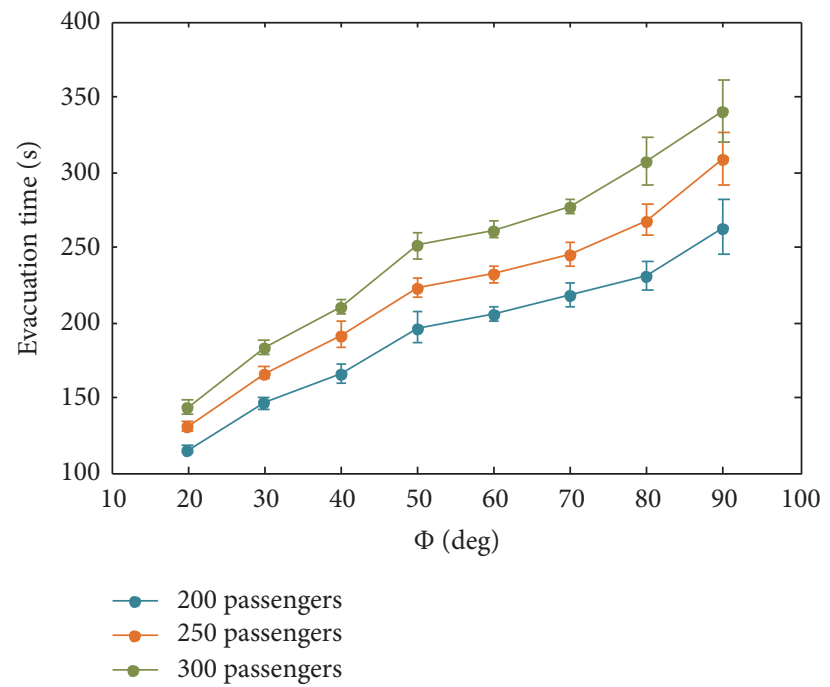

FIGURE 29: Evacuation times as a function of angle $\Phi$ in the Case 4 simulations.

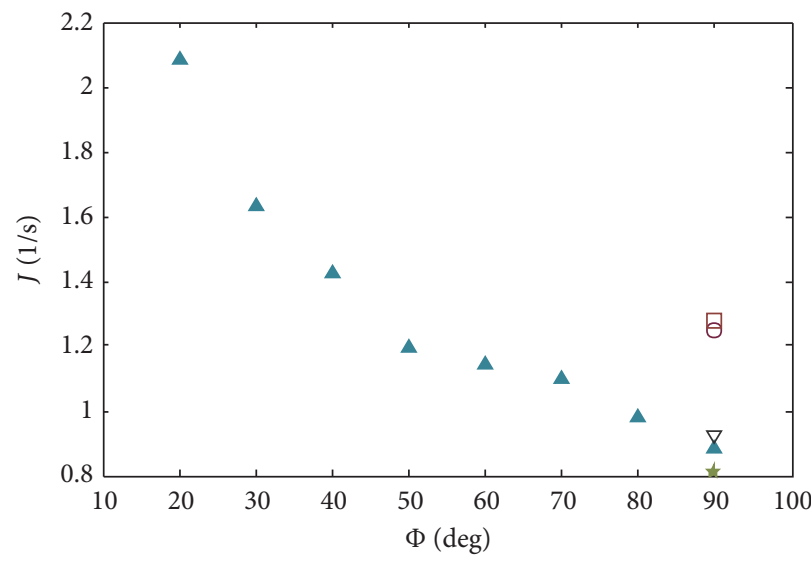
A The proposed
$\square$ Simulex
○ Seyfried
$\nabla$ MASSEgress
* FDS + Evac

FIGURE 30: Comparison of the outgoing flow with literature data. 


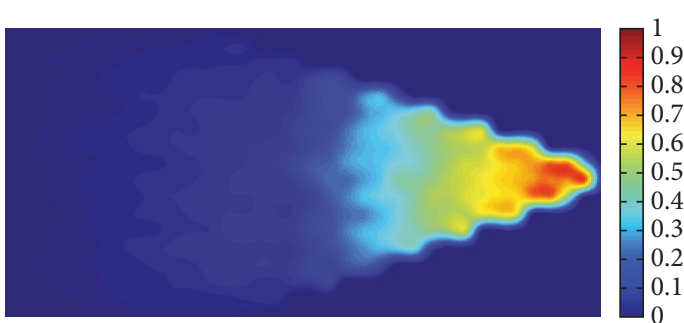

(a)

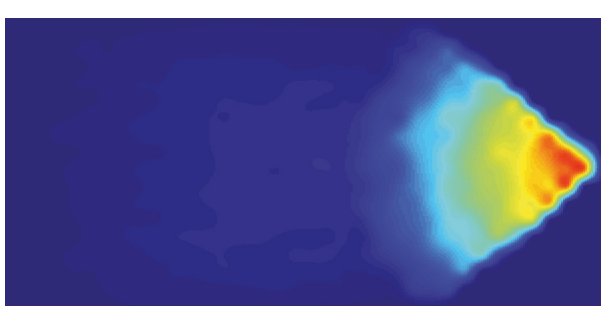

(c)

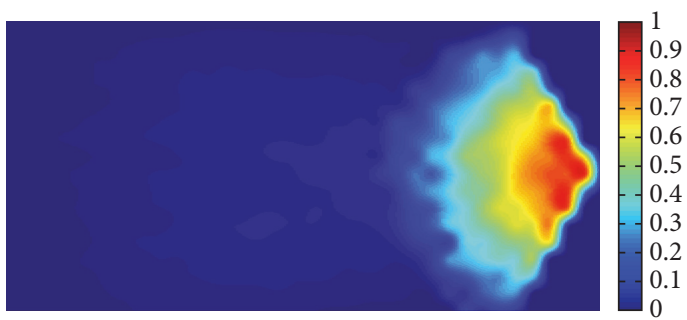

(e)

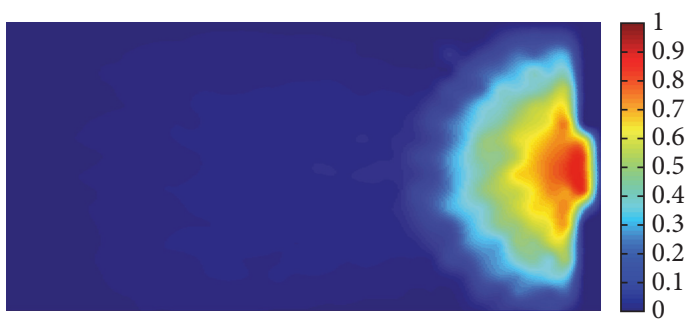

(g)

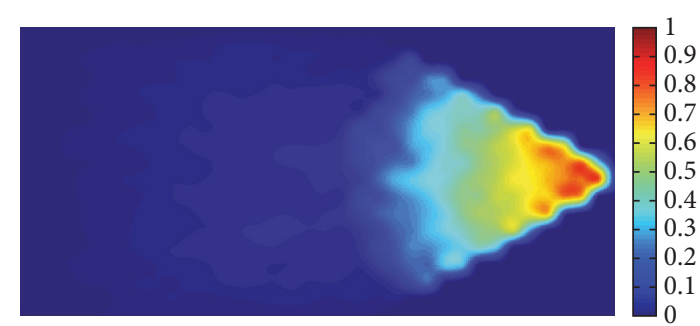

(b)

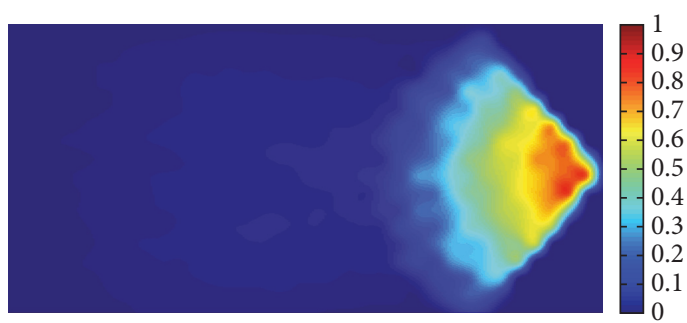

(d)

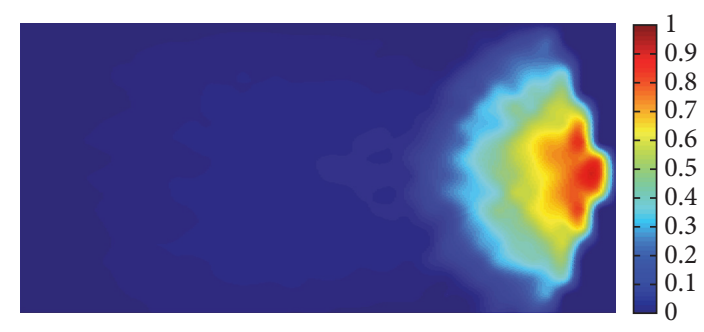

(f)

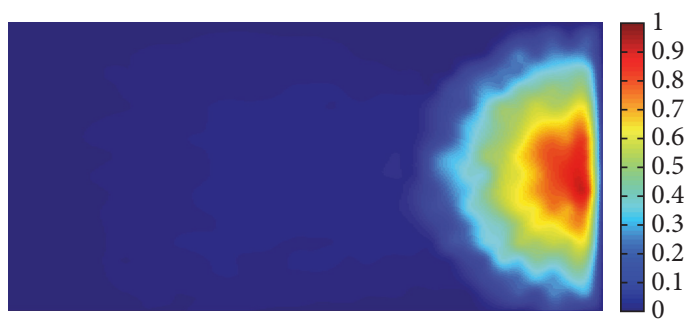

(h)

Figure 31: Space utilization diagram for Case 4; color bar indicating different occupied frequencies. (a) $\Phi=20^{\circ}$, (b) $\Phi=30^{\circ},(\mathrm{c}) \Phi=40^{\circ}$, (d) $\Phi=50^{\circ}$, (e) $\Phi=60^{\circ}$, (f) $\Phi=70^{\circ}$, (g) $\Phi=80^{\circ}$, and (h) $\Phi=90^{\circ}$.

4 walls that the passenger cannot traverse, and the resistance force associated with stools is added to the underlying movement model in order to impede the movement of passengers. We increased the cost of graph edges, which intersect the stools in order to endow the passenger with the behaviors of bypassing and crossing obstacles. Furthermore, we increased computational efficiency by decreasing the range of influence of the interaction force.

We conducted a series of evacuation simulations with different simulation cases. The simulations verify that the proposed model provides a valuable tool to evaluate the evacuation process with obstacles present in the cabins of a passenger ship. The results indicate that the funnel-shaped exits can improve the evacuation efficiency. It is important to also identify the potential negative influence of obstacles within the cabin and to provide useful suggestions for design of passenger ships.

However, the current model does not include all of the passenger behaviors and goals that arise in an actual evacuation. In a future work, we will consider more passenger behaviors, such as finding life jackets, choosing exits with which they are familiar, or changing their evacuation routes due to long wait times. In the current work, we only considered the impact of stools on evacuation; however, in some passenger ships, tables are equipped with chairs, and the influence of chairs on the movement of passengers 


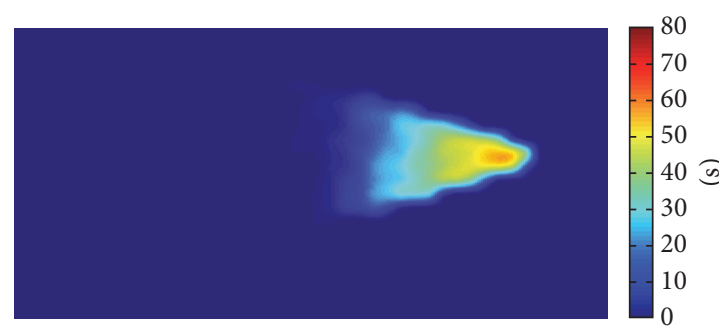

(a)

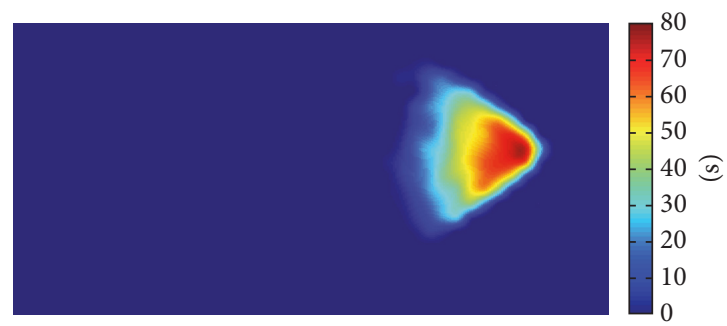

(c)

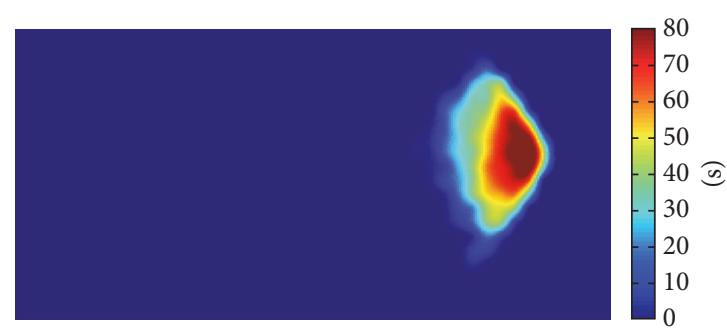

(e)

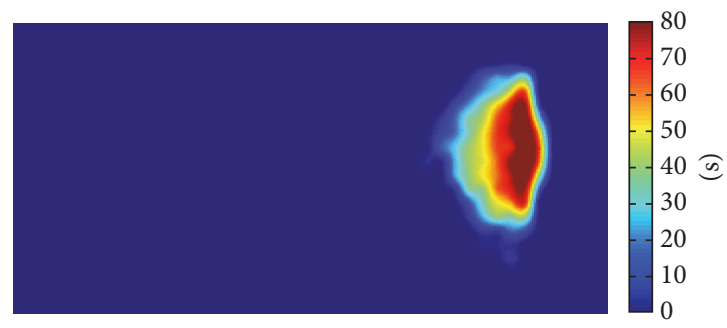

(g)

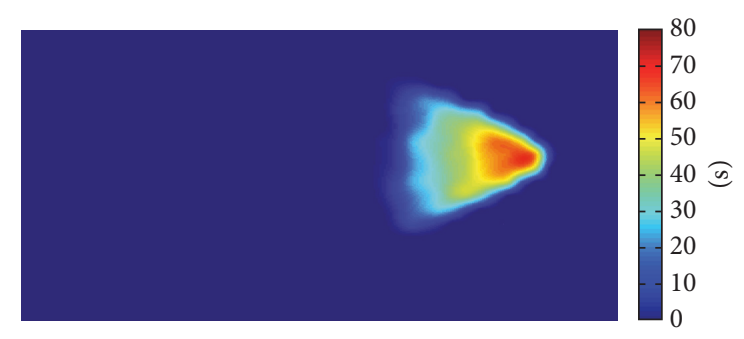

(b)

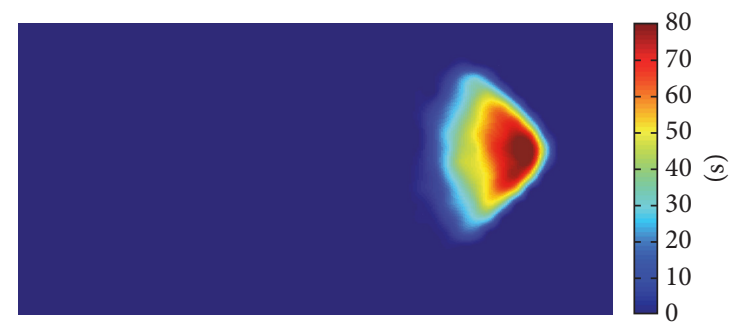

(d)

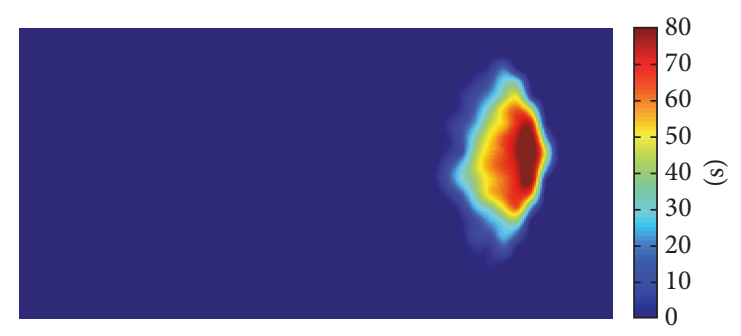

(f)

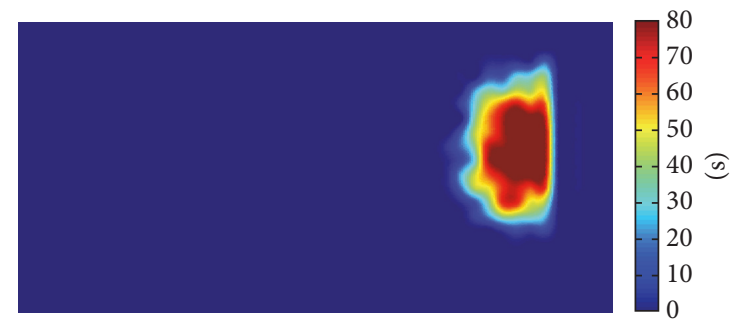

(h)

FIGURE 32: Cumulated density plot of occupant density exceeding the critical value $\left(3.5\right.$ persons $\left./ \mathrm{m}^{2}\right)$ for Case 4 ; color bar indicating different blocking time. (a) $\Phi=20^{\circ}$, (b) $\Phi=30^{\circ}$, (c) $\Phi=40^{\circ}$, (d) $\Phi=50^{\circ}$, (e) $\Phi=60^{\circ}$, (f) $\Phi=70^{\circ}$, (g) $\Phi=80^{\circ}$, and (h) $\Phi=90^{\circ}$.

also needs to be addressed in the future. Furthermore, real life evacuation experiments are also needed to validate the current model.

\section{Conflicts of Interest}

The authors declare that there are no conflicts of interest regarding the publication of this paper.

\section{Acknowledgments}

The study is supported by the National Natural Science Foundation of China (Grant no. 51409054), the National Science and Technology Major Project of the Ministry of Science and Technology of China (Grant no. Z12SJENA0014), and the Fundamental Research Funds for the Central Universities (Grant no. HEUCFJ170106).

\section{References}

[1] IMO, Guidelines for evacuation analysis for new and existing passenger ships, International Maritime Organization, 2007.

[2] W. Lei, A. Li, and R. Gao, "Effect of varying two key parameters in simulating evacuation for a dormitory in China," Physica A: Statistical Mechanics and its Applications, vol. 392, no. 1, pp. 7988, 2013.

[3] W. Lei, A. Li, R. Gao, N. Zhou, S. Mei, and Z. Tian, "Experimental study and numerical simulation of evacuation from a dormitory," Physica A: Statistical Mechanics and its Applications, vol. 391, no. 21, pp. 5189-5196, 2012. 
[4] L. Zhang, Y. Wang, H. Shi, and L. Zhang, "Modeling and analyzing 3D complex building interiors for effective evacuation simulations," Fire Safety Journal, vol. 53, pp. 1-12, 2012.

[5] M.-I. Roh and S. Ha, "Advanced ship evacuation analysis using a cell-based simulation model," Computers in Industry, vol. 64, no. 1, pp. 80-89, 2013.

[6] B. Ni, Z. Li, and X. Li, "Agent-based evacuation in passenger ships using a goal-driven decision-making model," Polish Maritime Research, vol. 24, no. 94, pp. 56-67, 2017.

[7] J. A. Capote, D. Alvear, O. Abreu, and A. Cuesta, "Analysis of evacuation procedures in high speed trains fires," Fire Safety Journal, vol. 49, pp. 35-46, 2012.

[8] J. A. Capote, D. Alvear, O. Abreu, A. Cuesta, and V. Alonso, "A Stochastic Approach for Simulating Human Behaviour During Evacuation Process in Passenger Trains," Fire Technology, vol. 48, no. 4, pp. 911-925, 2012.

[9] Z.-M. Fang, W. Lv, L.-X. Jiang, Q.-F. Xu, and W.-G. Song, "Modeling and assessment of civil aircraft evacuation based on finer-grid," Physica A: Statistical Mechanics and its Applications, vol. 448, pp. 102-112, 2016.

[10] S. Wolfram, "Statistical mechanics of cellular automata, Reviews of Modern Physics, vol. 55, no. 3, pp. 601-644, 1983.

[11] J. Ma, S. M. Lo, and W. G. Song, "Cellular automaton modeling approach for optimum ultra high-rise building evacuation design," Fire Safety Journal, vol. 54, pp. 57-66, 2012.

[12] W. Song, Y. Yu, W. Fan, and H. Zhang, "A cellular automata evacuation model considering friction and repulsion," Science in China Ser. E Engineering Materials Science, vol. 48, no. 4, pp. 403-413, 2005.

[13] L. A. Pereira, L. H. Duczmal, and F. R. B. Cruz, "Congested emergency evacuation of a population using a finite automata approach," Safety Science, vol. 51, no. 1, pp. 267-272, 2013.

[14] D. Helbing, I. Farkas, and T. Vicsek, "Simulating dynamical features of escape panic," Nature, vol. 407, no. 6803, pp. 487490, 2000.

[15] D. Helbing and P. Molnár, "Social force model for pedestrian dynamics," Physical Review E: Statistical, Nonlinear, and Soft Matter Physics, vol. 51, no. 5, pp. 4282-4286, 1995.

[16] D. Helbing, I. J. Farkas, P. Molnar, and T. Vicsek, "Simulation of pedestrian crowds in normal and evacuation situations," Pedestrian and Evacuation Dynamics, vol. 21, no. 2, pp. 21-58, 2002.

[17] J. K. K. Yuen and E. W. M. Lee, "The effect of overtaking behavior on unidirectional pedestrian flow," Safety Science, vol. 50, no. 8, pp. 1704-1714, 2012.

[18] F. Johansson, A. Peterson, and A. Tapani, "Waiting pedestrians in the social force model," Physica A: Statistical Mechanics and its Applications, vol. 419, pp. 95-107, 2015.

[19] S. Heliövaara, T. Korhonen, S. Hostikka, and H. Ehtamo, "Counterflow model for agent-based simulation of crowd dynamics," Building and Environment, vol. 48, no. 1, pp. 89-100, 2012.

[20] Y. Ma, R. K. K. Yuen, and E. W. M. Lee, "Effective leadership for crowd evacuation," Physica A: Statistical Mechanics and its Applications, vol. 450, pp. 333-341, 2016.

[21] E. Bonabeau, "Agent-based modeling: methods and techniques for simulating human systems," Proceedings of the National Acadamy of Sciences of the United States of America, vol. 99, no. 3, pp. 7280-7287, 2002.

[22] F. Tang and A. Ren, "GIS-based 3D evacuation simulation for indoor fire," Building and Environment, vol. 49, no. 1, pp. 193202, 2012.
[23] D. Chen, L. Wang, A. Zomaya et al., "Parallel simulation of complex evacuation scenarios with adaptive agent models," IEEE Transactions on Parallel and Distributed Systems, vol. 26, no. 3, pp. 847-857, 2014.

[24] A. Braun, B. E. Bodmann, and S. R. Musse, "Simulating virtual crowds in emergency situations," in Proceedings of the the ACM symposium on Virtual Reality Software and Technology VRST, p. 244, Monterey, Calif, USA, November 2005.

[25] J. Hu, L. You, J. Wei, M. Gu, and Y. Liang, "The effects of group and position vacancy on pedestrian evacuation flow model," Physics Letters A, vol. 378, no. 28-29, pp. 1913-1918, 2014.

[26] R. Lubaś, J. Wąs, and J. Porzycki, "Cellular Automata as the basis of effective and realistic agent-based models of crowd behavior," The Journal of Supercomputing, vol. 72, no. 6, pp. 2170-2196, 2016.

[27] E. Andresen, D. Haensel, M. Chraibi, and A. Seyfried, "Wayfinding and cognitive maps for pedestrian models," in Traffic and Granular Flow no. 15, pp. 249-256, 2016.

[28] B. Schröder, L. Arnold, A. Seyfried et al., "Knowledge-and Perception-based Route Choice Modelling in Case of Fire," in Proceedings of the Human Behaviour in Fire Symposium, September 2015.

[29] L. Crociani, A. Piazzoni, G. Vizzari, S. Bandini, C. Di Napoli, and S. Rossi, "When reactive agents are not enough: Tactical level decisions in pedestrian simulation," Intelligenza Artificiale, vol. 9, no. 2, pp. 163-177, 2015.

[30] L. Crociani, G. Vizzari, D. Yanagisawa, K. Nishinari, and S. Bandini, "Route choice in pedestrian simulation: Design and evaluation of a model based on empirical observations," Intelligenza Artificiale, vol. 10, no. 2, pp. 163-182, 2016.

[31] S. Ha, N.-K. Ku, M.-I. Roh, and K.-Y. Lee, "Cell-based evacuation simulation considering human behavior in a passenger ship," Ocean Engineering, vol. 53, pp. 138-152, 2012.

[32] K.-P. Park, S.-H. Ham, and S. Ha, "Validation of advanced evacuation analysis on passenger ships using experimental scenario and data of full-scale evacuation," Computers in Industry, vol. 71, article no. 2674, pp. 103-115, 2015.

[33] G.-N. Yuan, L.-N. Zhang, L.-Q. Liu, and K. Wang, "Passengers' evacuation in ships based on neighborhood particle swarm optimization," Mathematical Problems in Engineering, vol. 2014, Article ID 939723, 10 pages, 2014.

[34] M. Chen and D. Han, "Multi-Grid Model for Crowd's Evacuation in Ships Based on Cellular Automata," Polish Maritime Research, vol. 22, no. 1, pp. 75-81, 2015.

[35] Y. O. Cho, S. Ha, and K. P. Park, "Velocity-based egress model for the analysis of evacuation process on passenger ships," Journal of Marine Science and Technology, vol. 24, no. 3, pp. 466483, 2016.

[36] A. Varas, M. D. Cornejo, D. Mainemer et al., "Cellular automaton model for evacuation process with obstacles," Physica A: Statistical Mechanics and its Applications, vol. 382, no. 2, pp. 631642, 2007.

[37] R. Alizadeh, "A dynamic cellular automaton model for evacuation process with obstacles," Safety Science, vol. 49, no. 2, pp. 315-323, 2011.

[38] L. E. Aik and T. W. Choon, "Simulating evacuations with obstacles using a modified dynamic cellular automata model," Journal of Applied Mathematics, vol. 2012, Article ID 765270, 17 pages, 2012.

[39] M. J. Hurley, D. Gottuk, J. R. Hall et al., SFPE handbook of fire protection engineering, 5th edition, 2016. 
[40] V. M. Predtechenskii and A. I. Milinskii, "Planning for Foot Traffic Flow in Buildings NBS," 1978.

[41] S. P. Hoogendoorn and W. Daamen, "Pedestrian behavior at bottlenecks," Transportation Science, vol. 39, no. 2, pp. 147-159, 2005.

[42] S. J. Older, "Movement of pedestrians on footways in shopping streets," Traffic Engineering \& Control, vol. 10, no. 4, 1967.

[43] D. Helbing, A. Johansson, and H. Z. Al-Abideen, "Dynamics of crowd disasters: An Empirical Study," Physical Review E: Statistical, Nonlinear, and Soft Matter Physics, vol. 75, no. 4, Article ID 046109, 2007.

[44] C. W. Reynolds, "Steering behaviors for autonomous characters," in Proceedings of the Game Developers Conference, pp. 763782, 1999.

[45] Y. Kobayashi, T. Kaneda, M. Tamada, T. Shimura, and K. Hata, "Evacuation agent simulation in an underground shopping street adding a floor field approach and its three dimensional expression," in Pedestr. Evacuation Dyn, U. Weidmann, U. Kirsch, and M. Schreckenberg, Eds., pp. 673-681, Springer International Publishing, Cham, Switzerland, 2014.

[46] E. W. Dijkstra, "A note on two problems in connexion with graphs," Numerische Mathematik, vol. 1, pp. 269-271, 1959.

[47] S. J. Russell and P. Norvig, Artificial Intelligence: A Modern Approach, Prentice Hall, Upper Saddle River, NJ, USA, 3rd edition, 2010.

[48] A. Schadschneider, W. Klingsch, H. Kluepfel, T. Kretz, C. Rogsch, and A. Seyfried, "Evacuation dynamics: empirical results, modeling and applications," in Encyclopedia of Complexity and System Science, pp. 517-550, 2008.

[49] A. Seyfried, O. Passon, B. Steffen, M. Boltes, T. Rupprecht, and W. Klingsch, "New insights into pedestrian flow through bottlenecks," Transportation Science, vol. 43, no. 3, pp. 395-406, 2009.

[50] T. Korhonen and S. Heliövaara, "FDS+ Evac: Modelling Pedestrian Movement in Crowds," in Pedestr. Evacuation Dyn, pp. 823-826, Springer, 2011. 


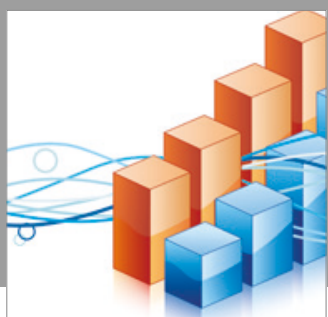

Advances in

Operations Research

vatersals

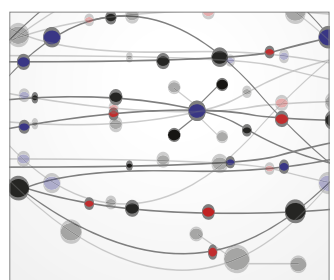

\section{The Scientific} World Journal
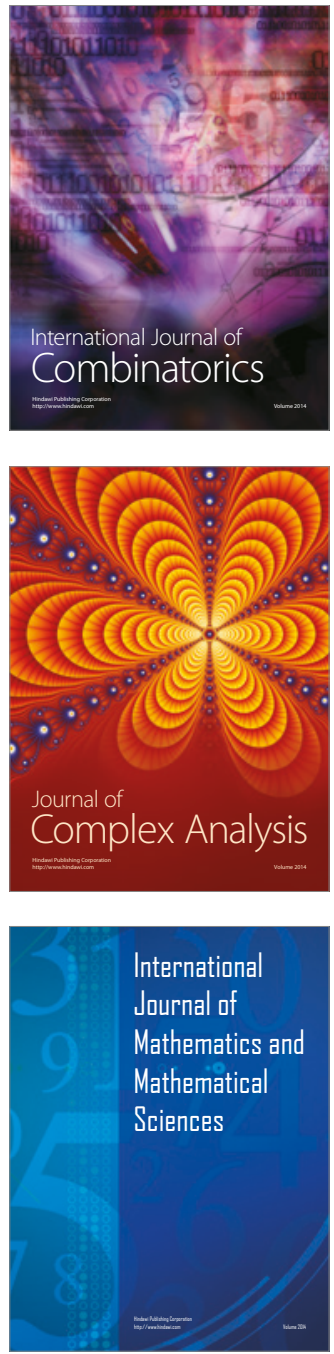
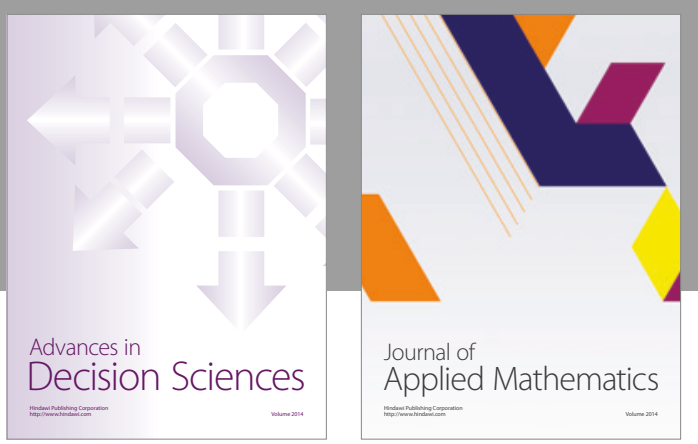

Algebra

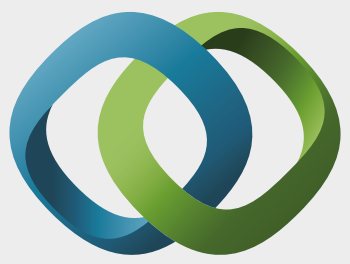

\section{Hindawi}

Submit your manuscripts at

https://www.hindawi.com
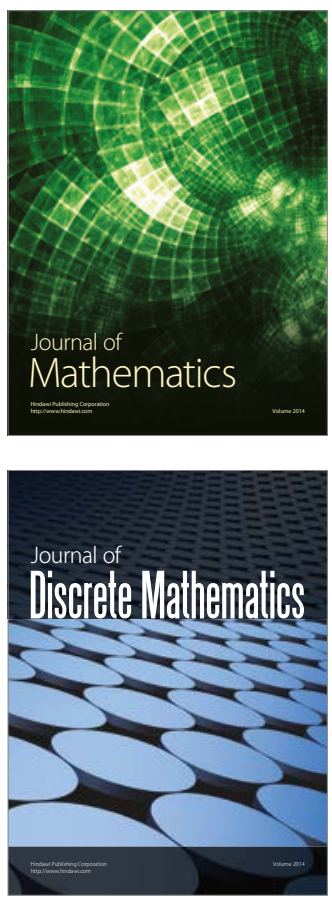

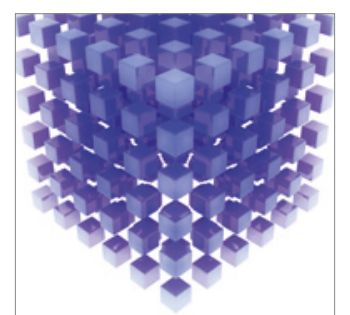

Mathematical Problems in Engineering
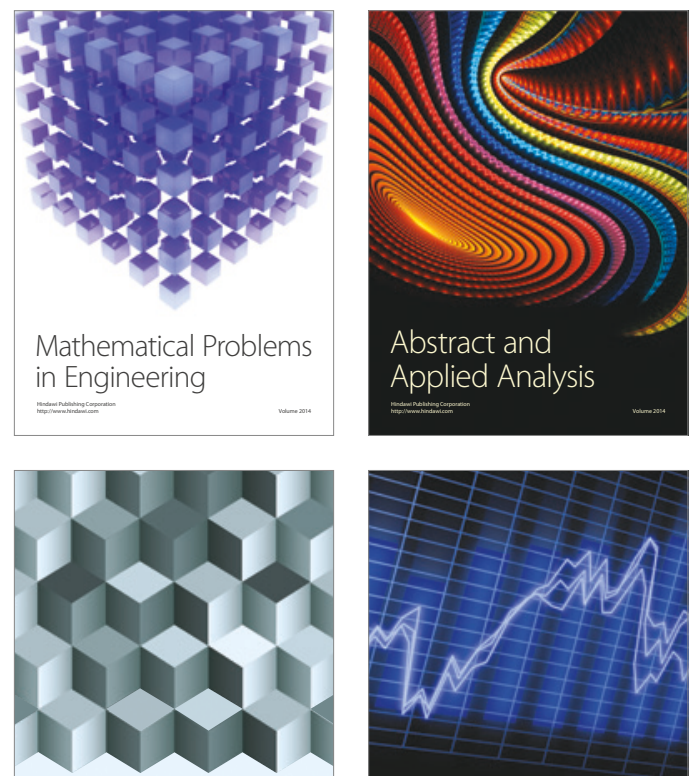

Journal of

Function Spaces

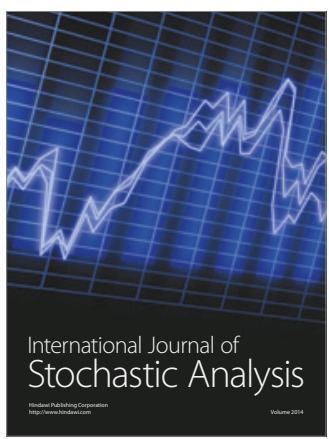

Probability and Statistics
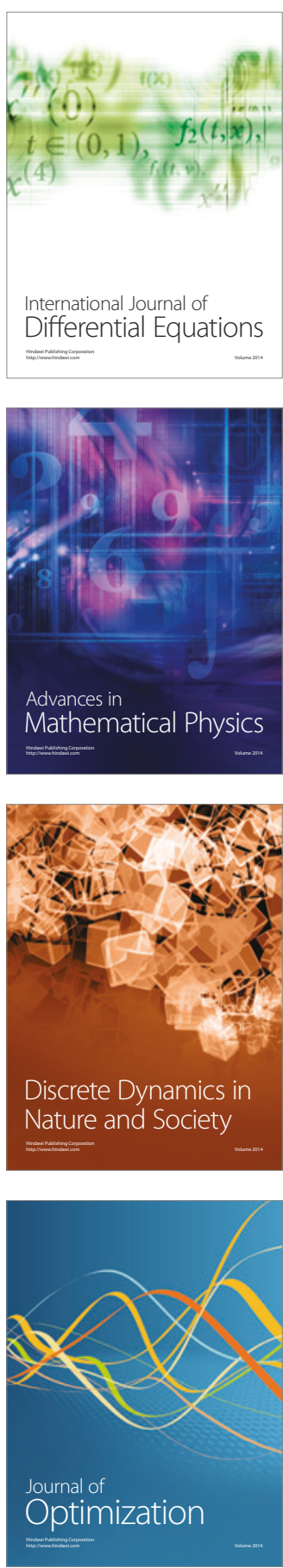